\title{
Path integration mediated systematic search: A Bayesian model
}

\author{
Robert J. Vickerstaff ${ }^{a, *}$, Tobias Merkle ${ }^{b}$ \\ a AgResearch Ltd, Lincoln Research Centre, Cnr Springs Road and Gerald Street, Private Bag 4749, Christchurch 8140, New Zealand \\ ${ }^{\mathrm{b}}$ Centre for Visual Sciences, Research School of Biology, Australian National University, Canberra ACT 2601, Australia
}

\section{H I G H L I G H T S}

- Path integration (PI) is a navigation strategy widely used in nature.

- Desert ants Cataglyphis fortis use PI while searching for their nest.

- We introduce a model of this search behaviour using Bayesian statistics.

- Performs favourably against three simpler models and similar to real ant searches.

\section{A R T I C L E I N F O}

\section{Article history:}

Received 8 December 2011

Received in revised form

26 April 2012

Accepted 28 April 2012

Available online 7 May 2012

Keywords:

Cataglyphis fortis

Navigation

Search theory

Bayesian search

Directed walk

\begin{abstract}
A B S T R A C T
The systematic search behaviour is a backup system that increases the chances of desert ants finding their nest entrance after foraging when the path integrator has failed to guide them home accurately enough. Here we present a mathematical model of the systematic search that is based on extensive behavioural studies in North African desert ants Cataglyphis fortis. First, a simple search heuristic utilising Bayesian inference and a probability density function is developed. This model, which optimises the short-term nest detection probability, is then compared to three simpler search heuristics and to recorded search patterns of Cataglyphis ants. To compare the different searches a method to quantify search efficiency is established as well as an estimate of the error rate in the ants' path integrator. We demonstrate that the Bayesian search heuristic is able to automatically adapt to increasing levels of positional uncertainty to produce broader search patterns, just as desert ants do, and that it outperforms the three other search heuristics tested. The searches produced by it are also arguably the most similar in appearance to the ant's searches.
\end{abstract}

(c) 2012 Elsevier Ltd. All rights reserved.

\section{Introduction}

This paper introduces a Bayesian-statistical (Ellison, 2004) model of target search where the target being searched for is a small, non-moving object such as a nest entrance and where the searching animal is using path integration (PI) as its only means of navigation. PI, also known as dead reckoning, is a process involving the continual integration of motion cues in order to maintain a running estimate of position and is widely observed in the animal kingdom (Mittelstaedt and Mittelstaedt, 1982; Collett and Collett, 2000; Gallistel, 1990; Redish, 1999; Wehner and Srinivasan, 2003; Etienne and Jeffery, 2004). We outline the relation to existing target search models from the search and rescue literature, and to existing search and PI models in biology. The model presented is intended to be specifically applicable to the nest search behaviour of desert ants, Cataglyphis fortis, a

\footnotetext{
* Corresponding author.

E-mail address: robert.vickerstaff@gmail.com (R.J. Vickerstaff).
}

species for which the mathematically convenient assumptions of a featureless environment, reliance on PI and the need to find a small, inconspicuous target (the nest entrance) are very appropriate (Wehner, 2003). Given the available data from this species, we take a quantitative comparison with our model as far as is feasible, highlighting the need for further data to validate modelling assumptions and parameter values. Since the model is relatively generic in nature, variations in the modelling assumptions and parameters would likely yield a model applicable to other ecological contexts.

Here we introduce a Bayesian model combining target search and PI. Given the inherent property of PI to accumulate positional error, the animal experiences cumulative uncertainty about its own position during the search. Since we assume less than perfect sensory input, it also experiences uncertainty about detection of the target. Hence the target, although stationary and at a known set of geocentric coordinates, may have already been encountered but not detected. We present a detailed Bayesian statistical model of the animal's state of knowledge about the error in its PI system during the search in the form of a probability density function 
(PDF) of the positional error. This PDF is then used to implement a simple search heuristic where the animal chooses its movement for the next time step to maximise the probability of target detection. This results in a search behaviour that outperforms three other examined search strategies, automatically adapts to changes in conditions (specifically, the PI error rate) and appears to resemble the search patterns of $C$. fortis reasonably well. Existing models of PI-mediated biological search (Wehner and Srinivasan, 1981; Müller and Wehner, 1994) have not incorporated such a detailed Bayesian treatment of the task and, conversely, the extensive Bayesian search literature does not generally make the assumption that the search platform is subject to the significant, cumulative positional uncertainty associated with biological PI, since more reliable positioning methods are generally available in a search and rescue situation, such as periodic satellite fixes (Richardson and Stone, 1971), and it is the target and not the searcher that is lost.

Bayesian statistical methods use a different definition of probability than the alternative frequentist interpretation (Ellison, 2004). Frequentist probabilities express the relative frequency of events, whereas Bayesian probabilities express the degree of belief in hypotheses. Frequentist inference gives the probability of observing the data if a given hypothesis is true, whereas Bayesian inference gives the probability of a given hypothesis being true given the observed data and relevant prior knowledge. The subjective nature of the definition of probability and the ability to incorporate prior knowledge make the Bayesian approach suitable for modelling the continuously changing state of knowledge of an animal about its position as it navigates. By using Bayesian inference it is possible to construct a model where the animal is assumed to retain all available information about its positional error in the form of a PDF, representing, as it were, a perfect memory of its past experience (the higher the PI error rate the lower the spatial resolution of the PDF required to retain all useful information). This is not sufficient to ensure an optimal search behaviour, since this would also require the animal to think ahead and make a perfect search plan using the PDF, whereas the present model only plans one movement at a time. Ideally, an optimal search strategy would be calculated, assuming perfect memory and perfect planning, under the constraints of noisy biological PI, and a comparison would reveal how closely an animal's search strategy approaches optimality. It seems unlikely that an ant's brain can explicitly implement such a sophisticated mechanism, but under natural conditions a much simpler search heuristic may be able to closely approximate it in efficiency. Exactly what a minimal mechanism for approximating an optimal search would be most likely depends on a range of factors, such as the source and severity of PI errors, the availability and reliability of nest detection cues and so on. The two extreme cases are illustrative: if navigation errors are minimal and nest detection is highly reliable, an optimal search is a simple Archimedean spiral requiring neither complex memory nor planning, whereas if navigation errors are extremely high, the animal is immediately lost and there is little ability to impose any coherent search strategy. In both cases a simple mechanism achieves the same efficiency as a sophisticated search, but must be selected based on the conditions whereas an optimal search adjusts itself to the conditions. There are similarities to the way in which cognitive maps (Tolman, 1948) (where landmarks are learned with associated positional information) are able to support efficient navigation behaviour, but in practise simpler mechanisms are sufficient to explain many observed behaviours, particularly in insects (Collett and Collett, 2006, 2009; Cruse and Wehner, 2011).

Searching for the small nest entrance in the desert ground is the crucial final component of the foraging trips of Cataglyphis ants. This remarkable animal has been shown to use the method of PI to navigate in its often flat and featureless environment.
The continual integration of motion cues (directions steered and distances covered) along its trajectory allows it to maintain a running estimate of its location relative to its nest entrance, often referred to as the home vector (HV), thereby enabling it to return close to the nest after a foraging excursion (Wehner, 2003; Vickerstaff and Di Paolo, 2005; Merkle et al., 2006a). PI is however subject to the gradual accumulation of errors (Wehner and Wehner, 1986; Müller and Wehner, 1988; Merkle et al., 2006b; Merkle and Wehner, 2009a) which become greater the longer the excursion has been (Merkle et al., 2006b; Merkle and Wehner, 2010). Therefore PI alone may not be sufficient to lead the ant back to the exact location of the nest entrance. Hence, the ant will begin a specific behaviour, the systematic search, that is characterised by a series of ever expanding loops centred upon its best initial estimate of the nest location - to which it will return at regular intervals - until the entrance is found (Wehner and Srinivasan, 1981; Müller and Wehner, 1994). These regular returns to the starting position of the search imply that PI is active during searching as well (Müller and Wehner, 1994). Overall, this distinctive search pattern increases the ants' chances of finding the nest entrance and thus surviving in the harsh desert environment (Harkness and Wehner, 1977; Schmid-Hempel and Schmid-Hempel, 1984). The overall searching density produced during this behaviour is approximately a radially symmetrical Gaussian bell shape and has been hypothesised to be a highly efficient search strategy given the limitations of biological PI (Wehner and Srinivasan, 1981) although it does not follow that the optimal search density should be equal to the initial PDF of the target's position (Hoffmann, 1983a). Since PI continues to be used to control the distribution of searching effort over space, the ants are still subjected to the gradual accumulation of PI positional uncertainty. Therefore, the most obvious search strategy, an Archimedean spiral, is certainly not the most effective strategy since navigation errors or nest detection failure could lead the ant to miss the nest on the first encounter, and an ever expanding spiral would never bring it back there (Hoffmann, 1983a).

Recently, it has became evident that the searching behaviour of desert ants is very flexible and adaptive (Merkle et al., 2006b; Merkle and Wehner, 2009b, 2010). Ants take into account the characteristics of the preceding foraging excursion by adjusting the extension of their search patterns: the further they have ventured out from the nest and the larger the error they have accumulated during their trip the wider their search from the beginning (Merkle et al., 2006b; Merkle and Wehner, 2010). That is, they calibrate their search according to the increasing uncertainty in their PI system. Not only, however, do they account for this but they also make use of additional cues along the route or close to the nest in order to increase their efficiency in searching for the nest (Merkle and Wehner, 2009b). While the search patterns of other central-place foraging insects look very similar to the search patterns of Cataglyphis ants (e.g. ants, Fourcassie and Traniello (1994); desert isopods, Hoffmann (1983a, 1983b); cockroaches, Durier and Rivault (1999)), it is still unclear whether these species are as adaptive in their search behaviour.

Completing their foraging trips and performing their search for the nest entrance, desert ants are faced with several uncertainties: the distance at which they detect their nest is quite short (approx. 2-3 cm, personal observation Merkle) and may be comparable to the very short, $2 \mathrm{~cm}$, detection range of desert isopods reported by Hoffmann (1983a, 1983b). Even this ability is not completely reliable either as ants occasionally fail to detect the entrance even when within this distance. Similarly, there are major uncertainties in PI as errors accumulate during the home run - the reason for the systematic search in the first place (see above) - but also during the search itself (Müller and 
Wehner, 1994; Merkle and Wehner, 2010). PI-related errors can arise from different subcategories: sensory (compass and odometer) errors (e.g., Wehner and Wehner, 1986; Wehner and Müller, 2006), errors in executing the relevant motor commands (for example at high walking speeds, see, Delcomyn and Usherwood, 1973) and errors in internally calculating position (PI per se, see, for instance, Müller and Wehner, 1988; Hartmann and Wehner, 1995).

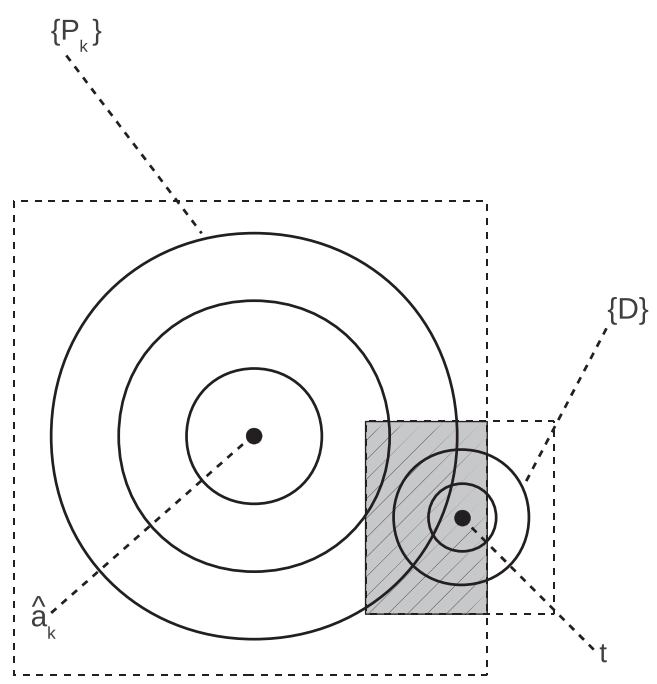

Fig. 1. Representation of the accelerated method used to calculate the estimated detection probability $\hat{s}_{k}$ (see Section 2.7). The PDF matrix, $\left\{\mathbf{P}_{\mathbf{k}}\right\}$, of the PI error is shown centred on the animal's estimated position, $\hat{\mathbf{a}}_{\mathbf{k}}$. The matrix form of the detection probability, $\{\mathbf{D}\}$, is shown centred on the position of the target, $\mathbf{t}$. The dot product of the shaded overlapping region gives the overall estimated detection probability $\hat{s}_{k}$. The box outlines represent the spatial extent of the two matrices. The concentric circles represent gradients within the probability values.
The question of just how optimal the characteristic search behaviour is remains unanswered and it is in fact a difficult one. The first step to tackle it is to evaluate the relevant sensory and neural capabilities that influence both the execution and success rate of the search. The questions that would have to be discussed in this regard are:

(1) How reliable is nest detection and over what range?

(2) At what rate and to what extent do the different types of errors accumulate and thus contribute to the overall error?

(3) Could it be possible that the ants use other cues to locate the nest entrance?

As to question 3, because of the flat, featureless terrain commonly inhabited by C. fortis (Dillier and Wehner, 2004), one could assume that virtually no usable landmarks or any other cues are at the ants' disposal for localisation other than their skylight compass and odometer. It has been demonstrated though that they are able to use, for instance, soil structure (Seidl and Wehner, 2006; Merkle, 2009) as a cue and it is also a possibility that they make use of the presence of nest mates to locate the nest entrance (Merkle, personal observation). These cues, however, do not seem to be sufficient as a backup solution to guide the animals to the nest entrance after the path integrator has failed to do so. Moreover, in the most common experimental paradigms ants are displaced to an unfamiliar test ground (see, for instance, Merkle et al., 2006b; Wehner and Wehner, 1990; Wehner et al., 2002); therefore the search is performed in an area guaranteed to be devoid of familiar landmarks. Previous studies were based on search patterns recorded in ants that had returned to the vicinity of the nest entrance but had not entered it. It is now known that such ants have not reset their path integrator to zero, a process which actually requires entering the nest (Knaden and Wehner, 2006). They were therefore beginning their search pattern with the PI error accumulated from the a

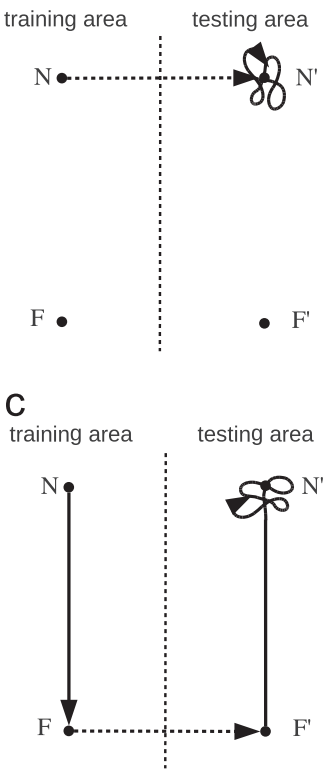

b

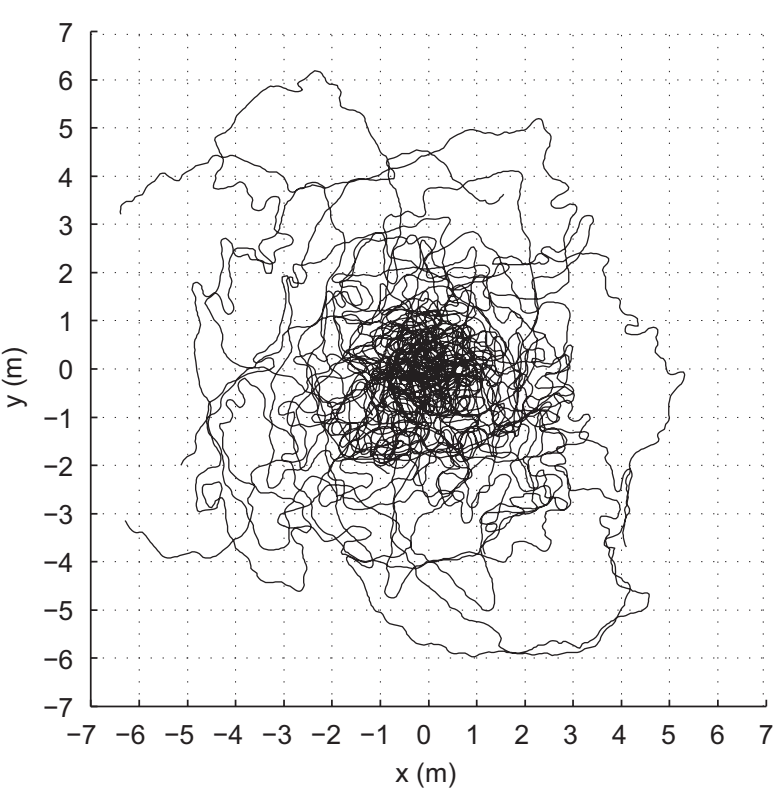

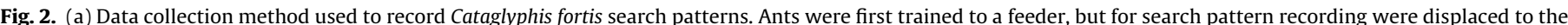

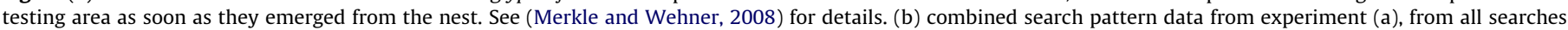

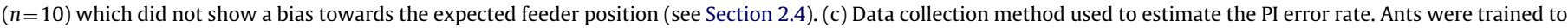

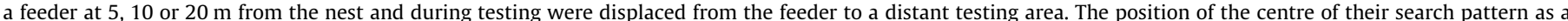

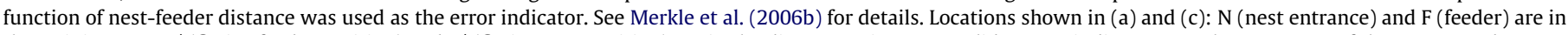

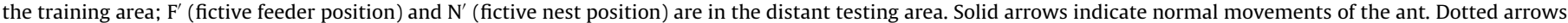
indicate passive displacements of the ant by the experimenter. 
preceding journey still present. In our approach, we instead use searches recorded from ants which have just emerged from the nest (Merkle and Wehner, 2008) (see Fig. 2 and Section 2.4 for further details), and can therefore be more confident that the PI system is zeroed at the start of search.

\section{Methods}

\subsection{Comparison to other biological models}

Nest search for a central-place forager is usually a part of its foraging behaviour. The need to return to the nest acts to anchor foraging to a specific location, unlike species which wander freely in search of food. Free wandering species are known to use a flexible combination of Brownian motion and Lévy flights depending on prey density (Humphries et al., 2010). A variant of the Lévy flight model, called the Lévy loop model (Reynolds, 2008), exists to model central-place foraging, and will be implemented and compared in the performance to the present Bayesian model. Here we do not model food discovery in the ant, which has been treated elsewhere (see for example Harkness and Maroudas, 1985), but only the return of the animal to a familiar location after some preceding excursion. The environment is assumed to provide no cues pointing towards the target, therefore no behaviour resembling chemotaxis, such as one following faint odour plumes (Vergassola et al., 2007) is possible. Landmarks are assumed absent, hence any homing strategy linked to landmark recognition or snapshot matching (for example Cartwright and Collett, 1987; Lambrinos et al., 2000) is ruled out, as is any strategy combining information from multiple cues (Cheng et al., 2007). The task is simply to discover the target location as quickly as possible, and we assume no trade offs of any kind exist, such as trying to minimise exposure to predators, disguise the nest location or accomplish any other goal.

Several mathematical models have been developed that can accurately describe the process of PI and also factor in the errors as described by experimental set-ups (for a review, see Vickerstaff and Cheung, 2010), as well as model the integration of PI with other navigation strategies (Cruse and Wehner, 2011). The systematic search behaviour of several Cataglyphis species has been investigated and modelled by Wehner and Srinivasan (1981), who provided a detailed analysis of the search patterns. Müller and Wehner (1994) showed that the systematic search of $C$. fortis can be simulated by spiralling trajectories that are regularly reset and that become wider and wider. Whereas search patterns produced by this simple algorithm closely resembled those observed in C. fortis, they could not consider the high flexibility and adaptiveness of the search as discovered in recent experimental work (Merkle et al., 2006b; Merkle and Wehner, 2010). Hoffmann (1983a, 1983b) describes the systematic search behaviour of the desert isopod Hemilepistus reaumuri, which occurs when the animal has been displaced by the experimenter a short distance from its burrow and shows that the search behaviour is approximately as efficient as an optimal search plan calculated using search theory, and more efficient than any form of Brownian search. Hoffmann's analysis will be compared to the present modelling approach in more detail in the discussion.

Cruse and Wehner (2011) present a model where PI and landmark based navigation are combined in such a way as to replicate major features of insect navigation without using a cognitive map. The area concentrated search (systematic search in our terminology) part of the model is a simple implementation of search intended as a stand in for the more sophisticated search capabilities of real insects, and serves to illustrate the role played by search within the context of a more complete set of navigational abilities than is investigated in the present account. The search method incorporates a tendency for clockwise turns giving it the ability to generate search loops similar to desert ants.

Directed walk (DW) theory (Cheung et al., 2007, 2008; Cheung and Vickerstaff, 2010) provides a method to classify the way in which different biological navigation strategies accumulate errors, and is highly relevant to PI. A DW consists of an animal attempting to take a series of steps of equal length and in the same direction, referred to as the axis of intended locomotion. Given an allothetic (externally generated) directional cue, such as a skylight- or magnetic-compass, the walk will behave as an allothetic directed walk (ADW) whereby it accumulates a positional deviation from the intended path at a constant mean rate. If the animal lacks an allothetic compass, and only has idiothetic (internally generated) directional cues, such as vestibular signals, the walk will behave as an idiothetic directed walk (IDW), and accumulate positional deviation from the intended path at an accelerating rate the further it walks. The basic result of DW theory is that an ADW can continue to make indefinite progress along the axis of intended locomotion, whereas the expected progress of an IDW reaches an upper limit beyond which no further progress is made (Cheung et al., 2007, 2008). This is a result of the IDW having no way to reset the animal's orientation relative to an allothetic directional cue, and therefore the orientation of the animal eventually has a random orientation relative to the axis of intended locomotion, whereas an ADW is able to reorient after each step taken so that, even though directional errors are made every step, they do not accumulate. DW theory has also been extended to apply to the behaviour of a HV being updated by a biological PI system (Cheung and Vickerstaff, 2010). Here the directed walk is taking place in representational space within the animal's nervous system. The main result of DW theory applied to PI is that two features must be present in the PI system in order for it to be robust to noise by accumulating errors in the manner of an ADW rather than an IDW: it must have an allothetic compass cue available and the HV must be stored using an allocentric static vectorial representation (ASVR), of which a geocentric Cartesian HV is an example. On these (Cheung and Vickerstaff, 2010) and other, related (Vickerstaff and Cheung, 2010) theoretical grounds this type has been proposed as the most biologically plausible class of PI system and will be referred to below as a compass-ASVR PI system.

\subsection{Comparison to concepts found in search theory}

Formal search theory (Benkoski et al., 1991; Frost, 1999; Stone, 1989) is a vast subject, and a comprehensive review is well beyond the scope of the present account. Here we briefly compare some of the common assumptions and concepts found in search theory with the present biological search problem, motivated by the obvious similarity between the two search tasks, as already noted by other authors (Hoffmann, 1983a; Alt, 1995). We conclude that a certain class of search theoretic problem has a great deal of similarity of PI target search provided that a compassASVR class biological PI system is assumed. Lack of space precludes a detailed mathematical treatment here, but we now outline a suitable scheme for comparing the two model types.

Search theory includes models where the searcher tries to find a randomly moving target, for example (Dell and Eagle, 1996). If we could only have the randomness perturb the searcher's estimate of its own position instead of the target's true position, then we might have a ready-made model of a searcher accumulating PI errors whilst looking for a fixed target. In search theory the target's random motion is usually assumed to have the Markovian property whereby movements each time step are statistically independent of all others and directed walk (DW) 
theory also makes this same assumption. As a result of this shared assumption the noise tolerant compass-ASVR class of PI system is expected to accumulate errors in a way closely analogous to the way that a randomly moving Markovian target accumulates displacement from its starting position-i.e. at a constant mean rate, not an accelerating one.

With this similarity recognised it is straightforward to make the connection between PI target search and moving target search explicit by a simple change of variables. As outlined in Section 2.3, instead of adding the PI error to the animal's HV or position each time step, it can be subtracted from the target position, giving a notional randomly moving nest entrance and a notionally error free PI system without changing the statistical behaviour of the model. This change does not influence the outcome at all since only the relative position of the target with respect to the searcher is important for determining when the target is found. A remaining difference in our model is that we assume two sources of error rather than one: errors recording intended movements and errors physically executing them. With this caveat in mind, we can consider moving target problems in search theory to be of considerable similarity to PI-mediated target search, and likely make use of results derived from the former.

A key dichotomy in search theoretic models is their formulation as either optimal search density problems or optimal search path problems (Benkoski et al., 1991). If the target's motion is slow or zero compared to the searcher's, corresponding in our case to a low or zero PI error rate, then the problem can be treated as a search density optimisation problem. If the target motion is comparable to the searcher's speed, the more difficult case of optimising the searcher's path must be solved, generally leading to a class of problems where optimal search paths are not guaranteed without exhaustive checking of all possible paths (Stone, 1989). For the latter case heuristic search methods have been developed such as (Dell and Eagle, 1996). In the absence of detailed data regarding the error rate, we have chosen to formulate the ant's search as a path optimisation problem, since the walking ant (unlike, say, a fast moving search and rescue vehicle) has no way of rapidly relocating its search without traversing the intervening ground. It is therefore appropriate to consider heuristic search optimisation methods rather than attempt to derive a truly optimal search strategy.

\subsection{Bayesian PI target search model}

Symbols in bold denote vectors or matrices. Symbols with a hat, $\hat{\mathbf{a}}$, indicate values estimated by the animal whereas plain symbols indicate exact values, whether known to the animal or not. See Tables 1 and 2 for a list of the mathematical symbols and abbreviations used in this account. The model is constructed using the assumption that the animal is aware of its own capabilities and limitations, such as the error rates and target detection probabilities. Variations in the model can easily be imagined where, for example, the animal operates using incorrect estimates of the true error rate parameters, or with uncertainty about how its ability to detect the target varies with distance, but such variations are beyond our present scope.

At time $k \in \mathbb{Z}$ an animal is at position $\mathbf{a}_{\mathbf{k}} \in \mathbb{R}^{2}$ within a continuous two dimensional space containing a target object at position $\mathbf{t} \in \mathbb{R}^{2}$. Each time step the animal chooses an intended movement, $\Delta \mathbf{a}_{\mathbf{k}+\mathbf{1}} \in \mathbb{R}^{2}$, with a maximum step length $\left|\Delta \mathbf{a}_{\mathbf{k}+\mathbf{1}}\right| \leq \lambda$, but when it executes the movement an additional unintended random movement, $\boldsymbol{\varepsilon}_{\mathbf{k}+\mathbf{1}}^{\text {exe }} \in \mathbb{R}^{2}$, also occurs:

$\mathbf{a}_{\mathbf{k}+\mathbf{1}}=\mathbf{a}_{\mathbf{k}}+\Delta \mathbf{a}_{\mathbf{k}+\mathbf{1}}+\boldsymbol{\varepsilon}_{\mathbf{k}+\mathbf{1}}^{\mathrm{exe}}$

We assume that the movement execution error is drawn from a radially symmetrical bivariate Gaussian distribution with zero mean and a variance of $\sigma_{\text {exe }}^{2}$, i.e. $\varepsilon_{\mathbf{k}}^{\text {exe }} \sim \mathbf{N}\left(\mathbf{v}_{\mathbf{0}}, \sigma_{\text {exe }}^{2} \mathbf{v}_{\mathbf{1}}\right)$ where $\mathbf{v}_{\mathbf{0}}=\left[\begin{array}{ll}0 & 0\end{array}\right]^{T}, \mathbf{v}_{\mathbf{1}}=\left[\begin{array}{ll}1 & 1\end{array}\right]^{T}$. (To relate this formulation of PI target search to the search theoretic model with a randomly moving target, consider the animal's movements to be executed without error and a moving target at position $\overline{\mathbf{t}}_{\mathbf{k}+\mathbf{1}}=\overline{\mathbf{t}}_{\mathbf{k}}-\boldsymbol{\varepsilon}_{\mathbf{k}+\mathbf{1}}^{\text {exe }}$.) Using PI the animal simultaneously maintains an internal estimate of its position (i.e. a home vector $(\mathrm{HV})$ ), $\hat{\mathbf{a}}_{\mathbf{k}} \in \mathbb{R}^{2}$, which is similarly subject to a random error, $\boldsymbol{\varepsilon}_{\mathbf{k}+\mathbf{1}}^{\mathrm{rec}} \in \mathbb{R}^{2}$, each time step representing an error made by the PI system recording the movement just performed. If an efference copy of the motor command specifying the intended step $\Delta \mathbf{a}_{\mathbf{k}+\mathbf{1}}$ is assumed to drive PI the following relation results:

$\hat{\mathbf{a}}_{\mathbf{k}+\mathbf{1}}=\hat{\mathbf{a}}_{\mathbf{k}}+\Delta \mathbf{a}_{\mathbf{k}+\mathbf{1}}+\boldsymbol{\varepsilon}_{\mathbf{k}+\mathbf{1}}^{\mathrm{rec}}$

where $\boldsymbol{\varepsilon}_{\mathbf{k}}^{\text {rec }} \sim \mathbf{N}\left(\mathbf{v}_{\mathbf{0}}, \sigma_{\text {rec }}^{2} \mathbf{v}_{\mathbf{1}}\right)$. (A slightly different equation results if proprioceptive feedback is assumed instead of efference copy, but this possibility is here neglected for brevity.) By allowing moves to be executed in any direction without constraint we can neglect the orientation of the animal's body. This also implies that the execution and recording errors be independent of the movement direction. In SI units, the two error rate parameters, $\sigma_{\text {exe }}^{2}$ and $\sigma_{\text {rec }}^{2}$, are in units of $\mathrm{m}^{2} / \mathrm{s}$. The PI error, $\mathbf{e}_{\mathbf{k}}$, in the estimate of position is defined as the difference between the estimated and true positions as follows:

$\mathbf{e}_{\mathbf{k}}=\hat{\mathbf{a}}_{\mathbf{k}}-\mathbf{a}_{\mathbf{k}}$

Because the HV is recorded using geocentric Cartesian coordinates, the modelled PI system therefore falls within the ASVR class (Vickerstaff and Cheung, 2010; Cheung and Vickerstaff, 2010). In addition, although the animal's orientation is not explicitly defined, the model defined by Eqs. (1) and (2) implicitly assumes the animal has access to an allothetic directional cue, and hence the modelled system belongs to the noise tolerant compass-ASVR class. To see why the model implicitly assumes a compass, consider the animal performing a DW along the $x$ axis, i.e. $\Delta \mathbf{a}_{\mathbf{k}+\mathbf{1}}=\left[\begin{array}{ll}1 & 0\end{array}\right]^{T}, \forall k$. Errors occur executing and recording each step, and $\hat{\mathbf{a}}_{\mathbf{k}}$ drifts away from $\mathbf{a}_{\mathbf{k}}$, but the model provides no way for the mean direction of the physically executed step to drift relative to the mean direction of the recorded step, since the exact same vector $\Delta \mathbf{a}_{\mathbf{k}+\mathbf{1}}$ appears in both equations. Therefore the frames of reference must remain anchored, implying that the directional component of each error is erased each step. Were the animal's orientation to be explicitly modelled, then the more realistic and detailed error model employed by DW theory could be employed, where the animal makes a turning error and a step length error each movement, and the compass cue could be explicitly included or excluded as desired. However, this would significantly complicate the present model.

As long as the animal moves without gaining any additional positional information its PI error will increase in a straightforward manner as follows, assuming all errors are statistically independent:

$\mathbf{e}_{\mathbf{k}}=\mathbf{e}_{\mathbf{0}}+\sum_{i=1}^{k}\left(\varepsilon_{\mathbf{i}}^{\mathrm{rec}}-\varepsilon_{\mathbf{i}}^{\mathrm{exe}}\right)$

giving the following bivariate Gaussian PDF of the error after $k$ steps:

$\mathbf{e}_{\mathbf{k}} \sim \mathbf{N}\left(\mathbf{e}_{\mathbf{0}}, k \sigma_{\mathrm{err}}^{2} \mathbf{v}_{\mathbf{1}}\right)$

where $\sigma_{\text {err }}^{2}=\sigma_{\text {exe }}^{2}+\sigma_{\text {rec }}^{2}$.

Each time step the animal possesses a PDF, $\mathbf{P}_{\mathbf{k}}$, for the current value of the PI error $\mathbf{e}_{\mathbf{k}}$. $\mathbf{P}_{\mathbf{k}}$ is defined as a column vector giving the probability of each possible quantised value of $\mathbf{e}_{\mathbf{k}}$ :

$\mathbf{P}_{\mathbf{k}}=\left[p_{k 1} \ldots p_{k n_{z}}\right]^{T}$

$p_{k i}=p\left(\left[\mathbf{e}_{\mathbf{k}}\right]=\mathbf{z}_{\mathbf{i}}\right)$ 
Table 1

List of main mathematical symbols. Note that positions and error rates are usually expressed in SI units in the text, but always converted into units of PDF grid square widths for the simulation of the models, where one square width $=0.1 \mathrm{~m}$ and one simulation time step $=1 \mathrm{~s}$.

\begin{tabular}{|c|c|}
\hline Symbol & Meaning \\
\hline$k$ & Time \\
\hline $\mathbf{a}_{\mathbf{k}}$ & Animal's true position \\
\hline$\hat{\mathbf{a}}_{\mathbf{k}}$ & Animal's estimate of its true position \\
\hline $\mathbf{e}_{\mathbf{k}}$ & Error in animal's estimate of true position \\
\hline $\mathbf{t}$ & True position of the target being searched for \\
\hline$\Delta \mathbf{a}_{\mathbf{k}+\mathbf{1}}$ & Intended movement from $\mathbf{a}_{\mathbf{k}}$ to $\mathbf{a}_{\mathbf{k}+\mathbf{1}}$ \\
\hline$\lambda$ & Maximum length of intended movement (i.e. $\left|\mathbf{\Delta} \mathbf{a}_{\mathbf{k}+\mathbf{1}}\right|_{\max }$ ) \\
\hline$\varepsilon_{\mathbf{k}+\mathbf{1}}^{\text {exe }}$ & Error made executing movement from $\mathbf{a}_{\mathbf{k}}$ to $\mathbf{a}_{\mathbf{k}+\mathbf{1}}$ \\
\hline$\varepsilon_{\mathbf{k}+\mathbf{1}}^{\mathrm{rec}}$ & Error made recording movement from $\mathbf{a}_{\mathbf{k}}$ to $\mathbf{a}_{\mathbf{k}+\mathbf{1}}$ \\
\hline$\sigma_{\text {exe }}^{2}$ & Variance of movement execution error \\
\hline$\sigma_{\text {rec }}^{2}$ & Variance of movement recording error \\
\hline$\sigma_{\text {err }}^{2}$ & $\sigma_{\mathrm{exe}}^{2}+\sigma_{\mathrm{rec}}^{2}$ \\
\hline$\sigma_{\text {init }}^{2}$ & Variance of Gaussian positional uncertainty at start of search, usually $=n_{0} \sigma_{\mathrm{err}}^{2}$ \\
\hline $\mathbf{P}_{\mathbf{k}}$ & PDF of $\mathbf{e}_{\mathbf{k}}$ prior to trying to detect the target (consists of $p_{k i}$ ) \\
\hline$p_{k i}$ & Prior probability that $\mathbf{e}_{\mathbf{k}}$ lies within grid square $\mathbf{z}_{\mathbf{i}}$ \\
\hline $\mathbf{Q}_{\mathbf{k}}$ & PDF of $\mathbf{e}_{\mathbf{k}}$ after failing to detect the target (consists of $q_{k i}$ ) \\
\hline$q_{k i}$ & Posterior probability that $\mathbf{e}_{\mathbf{k}}$ lies within grid square $\mathbf{z}_{\mathbf{i}}$ \\
\hline$n_{z}$ & Total number of squares in the grid \\
\hline $\mathbf{z}_{\mathbf{i}}$ & Integer coordinates of a square in the PDF grid \\
\hline$s_{k}$ & Probability animal will detect target at time $k$ given not previously detected \\
\hline$\alpha$ & Probability of detecting the target at zero range \\
\hline$\beta$ & Range at which target detection has dropped to $\alpha / 2$ \\
\hline$\gamma$ & Parameter controlling how sharply the detection probability drops with distance \\
\hline $\mathbf{D}\left(\hat{\mathbf{a}}_{\mathbf{k}}\right)$ & Probability of target detection for each possible value of $\mathbf{e}_{\mathbf{k}}$ given $\hat{\mathbf{a}}_{\mathbf{k}}$ \\
\hline$d_{k i}$ & Element of $\mathbf{D}\left(\hat{\mathbf{a}}_{\mathbf{k}}\right)$, probability of target detection if $\mathbf{e}_{\mathbf{k}}$ lies within square $\mathbf{z}_{\mathbf{i}}$ \\
\hline$\hat{s}_{k}$ & Animal's estimate of probability of target detection \\
\hline \multirow[t]{2}{*}{$\mathbf{v}_{\mathbf{0}}$} & {$[0]$} \\
\hline & {$[0]$} \\
\hline \multirow[t]{2}{*}{$\mathbf{v}_{\mathbf{1}}$} & {$[1]$} \\
\hline & {$[1]$} \\
\hline$[\cdot]$ & Indicates rounding the position to the nearest grid square \\
\hline B( ) & Bayesian update function giving $\mathbf{Q}_{\mathbf{k}}$ from $\mathbf{P}_{\mathbf{k}}$ and $\mathbf{D}\left(\hat{\mathbf{a}}_{\mathbf{k}}\right)$ \\
\hline $\mathbf{T}$ & Transition probability matrix (consists of $\tau_{j i}$ ) \\
\hline$\tau_{j i}$ & Probability that $\left[\mathbf{e}_{\mathbf{k}+\mathbf{1}}\right]=\mathbf{z}_{\mathbf{j}}$ if $\left[\mathbf{e}_{\mathbf{k}}\right]=\mathbf{z}_{\mathbf{i}}$ given $\sigma_{\text {err }}^{2}$ \\
\hline $\mathbf{F}()$ & Function calculating the movement maximising target detection probability \\
\hline$n_{0}$ & Length of foraging excursion preceding search \\
\hline$n_{s}$ & Maximum time available for searching \\
\hline$w_{k}$ & Unconditional probability of target detection at time $k$ \\
\hline $\bar{w}_{k}$ & Cumulative probability target detected at or before time $k$ \\
\hline$w_{f}$ & Probability of failing to detect the target by the end of the search period \\
\hline$c_{f}$ & Cost of one failed search \\
\hline$\mu$ & Mean resource accumulation rate given search of length $n_{s}$ \\
\hline$\mu_{\max }$ & Mean resource accumulation for optimal length search $\leq n_{s}$ \\
\hline$p_{\text {centre }}$ & Anchored random search: probability moving towards centre rather than randomly \\
\hline$c_{\text {spiral }}$ & Spiral search: spiral in loops separated by $2 \pi c_{\text {spiral }}$ \\
\hline$l_{\min }, l_{\max }$ & Lévy loop search: minimum and maximum loop lengths \\
\hline$b$ & Lévy loop search: exponent parameter \\
\hline
\end{tabular}

Table 2

List of abbreviations.

\begin{tabular}{ll}
\hline Abbreviation & Meaning \\
\hline PI & Path integration (also known as vector navigation, dead reckoning) \\
PDF & Probability density function \\
PMF & Probability mass function \\
HV & Home vector (position of the animal with respect to the home location) \\
DW & Directed walk \\
ADW & Allothetic directed walk (walk made with an external directional reference) \\
IDW & Idiothetic directed walk (walk made without any external directional reference) \\
ASVR & Allocentric static vectorial representation (see text for meaning) \\
\hline
\end{tabular}

where [-] indicates the rounding of the vector to the nearest member of $\mathbb{Z}^{2}$ (however, note that the error itself is not actually rounded off in the model, otherwise the accumulation of errors of small magnitude would be prevented), and where $\mathbf{z}_{\mathbf{i}} \in \mathbb{Z}^{2}$ is a member of a set of size $n_{z}$ containing all the integer valued vectors within a square of suitable size centred about the origin (specifically, where the magnitudes of both coordinates of $\mathbf{z}_{\mathbf{i}}=\left[\begin{array}{ll}x_{i} & y_{i}\end{array}\right]^{T}$ are less than or equal to a maximum $e_{\max }$ chosen to include all 
values of $\left[\mathbf{e}_{\mathbf{k}}\right]$ encountered with significant probability during the simulation run giving a total of $\left.n_{z}=\left(2 e_{\max }+1\right)^{2}\right)$. Therefore $p_{k i}$ is the probability that the PI error lies somewhere within square $\mathbf{z}_{\mathbf{i}}$ at time $k$. During model execution the probabilities are normalised to sum exactly to unity: $\sum_{i=1}^{n_{z}} p_{k i}=1, \forall k$. After making $k$ steps, during which the target is not being searched for, the distribution of $\mathbf{e}_{\mathbf{k}}$ will be given by:

$p_{k i} \approx \frac{1}{2 \pi k \sigma_{\mathrm{err}}^{2}} \exp \left(-\frac{\left|\mathbf{z}_{\mathbf{i}}\right|^{2}}{2 k \sigma_{\mathrm{err}}^{2}}\right)$

When the animal begins searching for the target it tries to detect the target from its current position at the start of each time step. The probability of successful detection, $s_{k}$, is defined as a function of the distance between the animal's position, $\mathbf{a}_{\mathbf{k}}$, and the target, $\mathbf{t}$ :

$s_{k}=\frac{\alpha}{1.0+\exp \left(-\gamma\left(1.0-\frac{\left|\mathbf{a}_{\mathbf{k}}-\mathbf{t}\right|}{\beta}\right)\right)}$

where the detection probability is assumed to saturate at the value $\alpha$ at close range, and to drop to zero beyond a threshold distance $\beta$, with $\gamma$ controlling how sharp the drop in probability is. In the absence of any detailed experimental data concerning this function for $C$. fortis, this generic sigmoidal detection function was chosen since, because it does not decay slowly with distance, this allows more rapid model execution. We note however that the proper determination of this function, or at least a 'sweep width' parameter characterising it, is considered to be very important in the search theory literature (Frost, 1999), and will obviously effect search efficiency. The assumption that detection is directionally invariant, inevitable in a model neglecting the animal's orientation, also may not strictly hold true for the ant. Each time step a column vector, $\mathbf{D}\left(\hat{\mathbf{a}}_{\mathbf{k}}\right)$, is calculated as a function of the current $\mathrm{HV}$, containing the probability of target detection for each possible quantised value of the current positional error, $\left[\mathbf{e}_{\mathbf{k}}\right]$. The detection probability depends on the distance to the target, $\left|\mathbf{a}_{\mathbf{k}}-\mathbf{t}\right|$, which can be calculated by the animal using the relation $\mathbf{a}_{\mathbf{k}}-\mathbf{t}=\hat{\mathbf{a}}_{\mathbf{k}}-\mathbf{e}_{\mathbf{k}}-\mathbf{t} \approx\left[\hat{\mathbf{a}}_{\mathbf{k}}\right]-\left[\mathbf{e}_{\mathbf{k}}\right]-\mathbf{t}$ :

$$
\begin{aligned}
& \mathbf{D}\left(\hat{\mathbf{a}}_{\mathbf{k}}\right)=\left[d_{k 1}, \ldots d_{k n_{z}}\right]^{T} \\
& d_{k i}=p\left(\operatorname{detect} \mid\left[\mathbf{e}_{\mathbf{k}}\right]=\mathbf{z}_{\mathbf{i}}\right) \\
& \quad=\frac{\alpha}{1.0+\exp \left(-\gamma\left(1.0-\frac{\left|\left[\hat{\mathbf{a}}_{\mathbf{k}}\right]-\mathbf{z}_{\mathbf{i}}-t\right|}{\beta}\right)\right)}
\end{aligned}
$$

The animal's estimate of the probability that the target will be detected at time $k$ is:

$\hat{s}_{k}=\sum_{i=1}^{n_{z}} p_{k i} d_{k i}=\mathbf{P}_{\mathbf{k}} \cdot \mathbf{D}\left(\hat{\mathbf{a}}_{\mathbf{k}}\right)$

where . indicates the dot product operation. The search ends successfully as soon as the target is detected. Therefore to continue searching given that, with presumed probability $1-\hat{s}_{k}$, the target was not detected, the animal must Bayesian update the positional uncertainty. Given the prior $\operatorname{PDF}, \mathbf{P}_{\mathbf{k}}$, of the positional error, Bayesian inference is used to find the posterior PDF, $\mathbf{Q}_{\mathbf{k}}$, given that the target was not detected. Specifically, the probability of hypothesis $H_{k i}$, that $\left[\mathbf{e}_{\mathbf{k}}\right]=\mathbf{z}_{\mathbf{i}}$, is Bayesian updated given the evidence, $E_{k}$, that the target was not detected at time $k$ :

$$
\begin{aligned}
& \mathbf{Q}_{\mathbf{k}}=\left[q_{k 1} \ldots q_{k n_{z}}\right]^{T} \\
& H_{k i}:\left[\mathbf{e}_{\mathbf{k}}\right]=\mathbf{z}_{\mathbf{i}} \\
& p\left(E_{k} \mid H_{k i}\right)=1-d_{k i}
\end{aligned}
$$

$p\left(H_{k i}\right)=p_{k i}$

$p\left(E_{k}\right)=1-\hat{s}_{k}$

$p\left(H_{k i} \mid E_{k}\right)=\frac{p\left(H_{k i}\right) p\left(E_{k} \mid H_{k i}\right)}{p\left(E_{k}\right)}$

$q_{k i}=p_{k i}\left(1-d_{k i}\right) /\left(1-\hat{s}_{k}\right)$

The above Bayesian updating process will be expressed as a vector function $\mathbf{B}$ giving the posterior from the prior: $\mathbf{Q}_{\mathbf{k}}=\mathbf{B}\left(\mathbf{P}_{\mathbf{k}}, \mathbf{D}\left(\hat{\mathbf{a}}_{\mathbf{k}}\right)\right)$. Finally, $\mathbf{P}_{\mathbf{k}+\mathbf{1}}$, is found by matrix multiplication (our Matlab implementation performs this step in a more efficient way, as detailed in Section 2.7) of $\mathbf{Q}_{\mathbf{k}}$ with a transition probability matrix, $\mathbf{T}$, to reflect the increase in positional uncertainty that will result from taking another movement step and from updating the estimated position:

$$
\begin{aligned}
\mathbf{T} & =\left[\begin{array}{ccc}
\tau_{1,1} & \ldots & \tau_{1, n_{z}} \\
\vdots & \ddots & \\
\tau_{n_{z}, 1} & \ldots & \tau_{n_{z}, n_{z}}
\end{array}\right] \\
\tau_{j i} & =p\left(\left[\mathbf{e}_{\mathbf{k}+\mathbf{1}}\right]=\mathbf{z}_{\mathbf{j}} \mid\left[\mathbf{e}_{\mathbf{k}}\right]=\mathbf{z}_{\mathbf{i}}\right) \\
\tau_{j i} & \approx \frac{1}{2 \pi \sigma_{\mathrm{err}}^{2}} \exp \left(-\frac{\left|\mathbf{z}_{\mathbf{j}}-\mathbf{z}_{\mathbf{i}}\right|^{2}}{2 \sigma_{\mathrm{err}}^{2}}\right)
\end{aligned}
$$

$\mathbf{P}_{\mathbf{k}+\mathbf{1}}=\mathbf{T Q}_{\mathbf{k}}$

At each timestep the animal must choose which movement command, $\Delta \mathbf{a}_{\mathbf{k}+\mathbf{1}}$, to issue. The commanded movement will be assumed limited to a maximum distance, $\lambda$, in any direction, $\phi \in[0,2 \pi]$. For the Bayesian search model the choice of movement can be considered as a vector function, $\Delta \mathbf{a}_{\mathbf{k}+\mathbf{1}}=\mathbf{F}\left(\hat{\mathbf{a}}_{\mathbf{k}}, \mathbf{P}_{\mathbf{k}+\mathbf{1}}\right)$, using the current HV and the updated PDF to choose the movement command. The function $\mathbf{F}$ will now be defined verbally. The function uses an approximate method of finding the movement that would take the animal to the location with the highest estimated probability, $\hat{s}_{k+1}^{\star}$, of detecting the target at the next time step:

$\hat{s}_{k+1}^{\star}=\mathbf{P}_{\mathbf{k}+\mathbf{1}} \cdot \mathbf{D}\left(\hat{\mathbf{a}}_{\mathbf{k}}+\Delta \mathbf{a}_{\mathbf{k}+\mathbf{1}}\right)$

$\hat{s}_{k+1}^{\star}$ is only an estimate of the true value of $\hat{s}_{k+1}$ (and hence a meta-estimate of $s_{k+1}$ ) since the animal's new estimated position $\hat{\mathbf{a}}_{\mathbf{k}+\mathbf{1}}$ will be effected by the recording error $\boldsymbol{\varepsilon}_{\mathbf{k}+\mathbf{1}}^{\text {rec }}$. Since, in order to approximate continuous motion, the simulation timestep is chosen to ensure a significant overlap between successive detection areas even for steps of the maximum length, in practise it is sufficient to assume a step of maximum length, $\lambda$, and choose only the direction $\phi$. First the number of candidate moves to be evaluated is chosen as $n_{\text {moves }}=\left\lceil c_{\text {density }} 2 \pi \lambda / 2 \beta\right\rceil$, where $c_{\text {density }}>0$ is a density parameter $\left(c_{\text {density }}=1.5\right.$ was chosen $)$ and $\lceil\cdot\rceil$ indicates rounding up to the nearest whole number. A random starting orientation, $\phi_{\text {ori }}$ is chosen. $n_{\text {moves }}$ candidate moves are generated starting from $\phi_{\text {ori }}$ separated by angular increments of $2 \pi / n_{\text {moves }}$. The move giving the highest value of $\hat{s}_{k+1}^{*}$ is selected. This algorithm is intended to approximate the selection of the direction giving the highest value of $\hat{s}_{k+1}^{*}$ without having to evaluate an excessively large number of candidate moves, since this part of the model represents a very significant part of the computational cost of running the model. The higher the value of $c_{\text {density }}$ the more densely are the candidate moves arranged around the circle of radius $\lambda$ centred on the animal's current position, where a value of $c_{\text {density }}=1$ would give the minimum number of evaluations such that the candidate position's detection functions just begin to overlap. If all moves give a value of zero, indicating the animal is off the edge of the current PDF, the animal instead moves directly towards the position $\mathbf{t}$, i.e. $\phi=\operatorname{atan} 2\left(-\hat{\mathbf{a}}_{\mathbf{k}}\right)$. This concludes the definition of the 
movement selection function $\mathbf{F}$. The Bayesian PI target search model can now be summarised as follows (repeating and combining the relevant equations from above for convenience):

$$
\begin{aligned}
& \mathbf{P}_{\mathbf{k}+\mathbf{1}}=\mathbf{T B}\left(\mathbf{P}_{\mathbf{k}}, \mathbf{D}\left(\hat{\mathbf{a}}_{\mathbf{k}}\right)\right) \\
& \hat{\mathbf{a}}_{\mathbf{k}+\mathbf{1}}=\hat{\mathbf{a}}_{\mathbf{k}}+\mathbf{F}\left(\hat{\mathbf{a}}_{\mathbf{k}}, \mathbf{P}_{\mathbf{k}+\mathbf{1}}\right)+\boldsymbol{\varepsilon}_{\mathbf{k}+\mathbf{1}}^{\mathrm{rec}} \\
& \mathbf{a}_{\mathbf{k}+\mathbf{1}}=\mathbf{a}_{\mathbf{k}}+\mathbf{F}\left(\hat{\mathbf{a}}_{\mathbf{k}}, \mathbf{P}_{\mathbf{k}+\mathbf{1}}\right)+\boldsymbol{\varepsilon}_{\mathbf{k}+\mathbf{1}}^{\mathrm{exe}}
\end{aligned}
$$

To run the model, suitable starting values of $\mathbf{a}_{\mathbf{0}}$, $\hat{\mathbf{a}}_{\mathbf{0}}$ and $\mathbf{P}_{\mathbf{0}}$ must be chosen. For the purposes of simulating an ant searching for its nest at the end of a foraging excursion, time $k=0$ will be defined to refer to the start of search, not the moment the ant originally left the nest. Position $\mathbf{t}$ refers to the true nest position. It will be assumed that the animal has just undertaken a foraging excursion of some given round trip distance during which the PI error, e, was accumulating at the same rate as will apply during the search. For a foraging excursion of $n_{0}$ time steps starting at the nest with no PI error Eq. (4) gives the PDF for the error at the start of search as:

$\mathbf{e}_{\mathbf{0}} \sim \mathbf{N}\left(\mathbf{v}_{\mathbf{0}}, \sigma_{\text {init }}^{2} \mathbf{v}_{\mathbf{1}}\right)$

where $\sigma_{\text {init }}^{2}=n_{0} \sigma_{\text {err }}^{2}$, from which a value can be sampled. Using Eq. (5) the values of $\mathbf{P}_{\mathbf{0}}$ can also be calculated. It will be assumed that the ant begins nest search only once it has reached the expected nest position, i.e. when $\hat{\mathbf{a}}=\mathbf{t}$. Therefore, $\hat{\mathbf{a}}_{\mathbf{0}}=\mathbf{t}$ and Eq. (3) implies $\mathbf{a}_{\mathbf{0}}=\hat{\mathbf{a}}_{\mathbf{0}}-\mathbf{e}_{\mathbf{0}}$.

Running the model produces a time series of $s_{k}$ values, giving the conditional probability that the animal detects the target at time $k$ given that detection has not occurred on any previous time step. The unconditional probability, $w_{k}$, of detection occurring at time step $k$ is calculated as follows:

$w_{k}=s_{k} \prod_{i=0}^{k-1}\left(1-s_{i}\right)$

The cumulative probability, $\bar{w}_{k}$, that detection occurred at or before time step $k$ is $\bar{w}_{k}=\sum_{i=0}^{k} w_{i}$. The probability, $w_{f}$, that, after a time limit of $n_{s}$ time steps the target has not been found is $w_{f}=1-\bar{w}_{n_{s}}$, i.e. this value is the probability of search failure given a definite time limit.

In this way a single run of the simulation provides the probability mass function (PMF) for the discrete time $k$ that the target is discovered. A single run effectively simulates a population of searchers experiencing different outcomes of target detection, but all experiencing the exact same initial PI error, per-step movement execution errors and per-step movement recording errors. Because detection is modelled as an all-ornothing event, those searchers that detect the target drop out of the population immediately having ended their search and those that do not all continue searching with the same state as each other $\left(\mathbf{P}_{\mathbf{k}}, \hat{\mathbf{a}}_{\mathbf{k}}\right.$ and $\left.\mathbf{a}_{\mathbf{k}}\right)$. The state is simply Bayesian updated to reflect the failure to detect the target at that moment. It is therefore straightforward to model variations in target detection as a population rather than explicitly simulate individual searches experiencing different detection outcomes. A single simulation run, however, provides only one sampling of the initial PI error, $\mathbf{e}_{\mathbf{0}}$ and one possible sequence of the per-step execution errors $\varepsilon_{\mathbf{k}}^{\text {exe }}$ and recording errors, $\varepsilon_{\mathbf{k}}^{\text {rec }}$. Multiple runs must therefore be performed resampling these error sources and the resulting values of $w_{k}$ averaged across samples in order to account for these sources of uncertainty. It is not straightforward to use a PDF describing a population of searchers experiencing different recording errors since each error gets incorporated into the HV, â, and therefore leads to attempted target detections at different perceived locations, and therefore to a population with different internal PDFs $\mathbf{P}_{\mathbf{k}}$.
Given the PMF of target discovery time an ecologically relevant measure of searching performance can be formulated. For the present case, an ant colony will be assumed to be attempting to accumulate food items as quickly as possible. Two alternative efficiency measures are (i) the mean rate of resource accumulation, assuming that each successful target detection corresponds to an ant carrying a food item to its nest, and (ii) the mean number of successful foraging trips per forager life time, assuming premature death upon a failed search. Both require the estimation of additional parameters. Here we develop (i) since it is considerably easier to calculate.

Assume a probability of $w_{k}$ that a search ends after exactly $\delta_{k}$ time steps including the foraging time of $n_{0}$ steps and the search time (a maximum of $n_{s}$ steps), and that a reward of $\rho_{k}$ is accrued to the nest therefore giving a reward rate of $\rho_{k} / \delta_{k}$ reward units per timestep. $k=1$ denotes the start of search and $\delta_{k}$ ranges from the minimum possible round-trip time $\delta_{1}=n_{0}$ (i.e. the duration is just the foraging time, with a search time of zero) to the maximum possible duration of $\delta_{n_{s}+1}=n_{0}+n_{s}$ (where the search time limit is reached). Considering a random timestep from a large sample of journeys, the reward rate of the timestep will be $\rho_{k} / \delta_{k}$ with a probability $w_{k} \delta_{k} / \sum_{j} w_{j} \delta_{j}$. Therefore the overall mean reward rate per timestep is $\sum_{k} w_{k} \rho_{k} / \sum_{j} w_{j} \delta_{j}$. To handle the case where the animal fails to find the target, assume that with probability $w_{f}$ after $\delta_{f}=n_{0}+n_{s}$ time steps the ant has a negligible probability of ever finding the nest and eventually dies, and that the nest therefore generates a new worker at some additional cost $c_{f}$ in order to maintain the forager population. Assuming all rewards $\rho_{k}=1$ and given $w_{f}=1-\sum_{k} w_{k}$, the mean reward rate will be:

$\mu=\frac{1-w_{f}-w_{f} c_{f}}{\sum_{j} w_{j} \delta_{j}+w_{f} \delta_{f}}$

This measure of efficiency has an advantage over $\bar{w}_{n_{s}}$ in that it takes account of how rapidly the target is located during search, and can therefore distinguish between two strategies where $w_{f}$ is low, but one succeeds sooner than the other on average. While $\bar{w}_{n_{s}}$ can only increase as $n_{s}$ increases, $\mu$ may start to decline when the chances of finding the nest become small enough, implying that it is no longer worth waiting for the ant to try to return home, but rather better the pay the cost of a new forager and begin a new foraging trip immediately. This seems appropriate for a central place foraging context where individual survival is less important than colony survival. Because $\mu$ can begin to decline for large $n_{s}$, it is useful to define a final efficiency measure, $\mu_{\max }$, equal to the maximum value obtained when $\mu$ is calculated for each timestep of a search as if $k=n_{s}$, i.e. as if each timestep were the search time limit.

\subsection{Three alternative search strategies}

The three other searching models are now introduced along with the methods used to process search patterns recorded in $C$. fortis ants. The three search models differ from the Bayesian method (model 1) in that they do not make use of the PDF of the PI error when deciding which search movement to make, nor is the PDF required to calculate their search performance, however, the PDF can still be meaningfully calculated in the same manner as for the Bayesian model and correctly shows the searcher's evolving state of uncertainty about the PI error.

Model 2: Anchored random movement. This method is controlled by a parameter, $p_{\text {centre }} \in[0,1]$. Each time step, with probability $1-p_{\text {centre }}$ the animal selects a movement of the maximum distance $\lambda$, in a random direction $\phi \in[0,2 \pi]$; or, with probability $p_{\text {centre }}$ selects the movement which takes it back towards the 
estimated target position (i.e. $\phi=\operatorname{atan} 2\left(-\hat{\mathbf{a}}_{\mathbf{k}}\right)$ ). Thus $p_{\text {centre }}=0$ gives a completely random walk, whereas $p_{\text {centre }}=1$ gives motor commands which act to maintain $\hat{\mathbf{a}}_{\mathbf{k}}=\mathbf{t}$.

Model 3: Archimedes' spiral. The animal spirals out from the location $\hat{\mathbf{a}}_{\mathbf{0}}=\mathbf{t}$ along an Archimedean spiral where the relationship between the polar coordinates, $r_{k}=\left|\hat{\mathbf{a}}_{\mathbf{k}}-\mathbf{t}\right|$ and $\theta_{k}=\operatorname{atan} 2\left(\hat{\mathbf{a}}_{\mathbf{k}}-\mathbf{t}\right)$ is determined by $r_{k}=c_{\text {spiral }} \theta_{k}$, with initial condition $\theta_{0}=0$. Each time step $\theta_{k}$ is incremented such that the spiral progresses $\lambda$ distance units. Parameter $c_{\text {spiral }}(\mathrm{m} / \mathrm{rad})$ gives a separation between successive loops of the spiral of $2 \pi c_{\text {spiral }}$. The optimal choice for $c_{\text {spiral }}$ will depend on the other parameters of the search problem. A spiral of the length required for a given search time and $\lambda$ is first calculated at suitable fixed increments of $\theta$ (using the formula for the total spiral length $0.5 c_{\text {spiral }}\left(\theta_{\text {total }} \sqrt{1+\theta_{\text {total }}^{2}}+\sinh ^{-1} \theta_{\text {total }}\right)$, fitted with cubic splines (using the Matlab 'spline' command), then resampled as a series of positions such that each successive intended movement is of length $\lambda$. In the absence of recording errors the movement command each time step would be $\Delta \mathbf{a}_{\mathbf{k}+\mathbf{1}}=r_{k+1}\left[\cos \left(\theta_{k+1}\right) \sin \left(\theta_{k+1}\right)\right]^{T}-\hat{\mathbf{a}}_{\mathbf{k}}$, i.e. the movement required to take the animal to the next position of the search pattern, however, due to recording errors, the current position $\hat{\mathbf{a}}_{\mathbf{k}}$ will not generally be equal to the precalculated position for time step $k$, and consequently a movement command calculated in this way may exceed the allowed distance $\lambda$. To strictly enforce $\lambda$, if $\left|\Delta \mathbf{a}_{\mathbf{k}+\mathbf{1}}\right|>\lambda$ the movement vector is scaled down so that $\left|\Delta \mathbf{a}_{\mathbf{k}+\mathbf{1}}\right|=\lambda$. This correction is also applied in the same way to search models 4 and 5 , which are also based on replaying a predefined search pattern in the presence of recording errors.

Model 4: Lévy loop search. The animal makes successive straight excursions (loops) from the origin $\mathbf{t}$ in uniformly distributed random directions but of lengths selected from a powerlaw probability distribution. At the end of each excursion it returns to the origin along the same path. The model is characterised by three parameters, $l_{\min }$ and $l_{\max }$, defining the minimum and maximum excursion length, and $1<b<3$, the exponent of the power-law probability distribution. For each excursion a loop of length $l$ is chosen with a probability $A l^{b}$ where $A=\left((b-1) l_{\min }^{b-1}\right) /\left(1-\left(l_{\max } / l_{\min }\right)^{1-b}\right)$. A search path is precalculated using this algorithm by stepping the position along the outbound and inbound legs of each loop at positions separated by $\lambda$, each loop length being extended to the nearest whole number of steps. The precalculated search is then replayed in the same manner as the spiral search.

Model 5: Ant search replay. This method replays a real $C$. fortis search pattern. The search patterns that were used for the replay were part of an experiment (Merkle and Wehner, 2008) in which a feeder was established $10 \mathrm{~m}$ south of one ant colony's nest entrance. The area where the nest and feeder were situated was free of landmarks. All ants had undergone intensive training (at least one day) and had performed similar numbers of foraging and homing trips before being tested. In the test situation (see Fig. 2a), trained ants $(n=25)$ were captured immediately after leaving the nest (so-called 0\%-out ants, Merkle and Wehner, 2008) and transferred to a remote, landmark free test area where a grid pattern had been marked out on the desert surface. In the test area, searches were recorded by hand for $300 \mathrm{~s}$ on graph paper. Later, the hand-recorded paths were digitised using a graphics tablet and GEDIT Graphics Editor and Run Analyser (Antonsen, 1995 ) to give a positional value at approximately $5 \mathrm{~s}$ intervals (for full details, see Merkle and Wehner, 2008). All further data processing of the digitised paths was performed using Matlab (Version 7.3.0.298, R2006b).

Displacement of these ants to the test area disturbed them such that most of them did not head towards the position of the feeder but started searching for the nest instead. Having just emerged from the latter, they had reset their path integrator (Knaden and Wehner, 2006), that is, they knew the position of the nest entrance with the minimum possible error. The search behaviour of this group of ants therefore was best suited to base the analysing and modelling work on rather than ants that had returned from a feeder. The latter would have accumulated a significant error in their path integrator (see introductory section), making it impossible to be confident of the nest position assumed by the ant at the start of its search. Under natural conditions disturbances may be caused by predation attempts (Harkness and Wehner, 1977; Schmid-Hempel and Schmid-Hempel, 1984) and therefore displaced outbound ants trying to return to the nest rather than continuing to forage may be employing an anti-predation response (Merkle and Wehner, 2008).

As the search patterns from the selected ants from this experiment could have still been biased towards the feeder position (to which the animals had been trained), we excluded all runs where the extension in one direction exceeded the extension in all other directions by at least $1 \mathrm{~m}$. In addition, all runs with median or mean $x$ - or $y$-values of $>1.5 \mathrm{~m}$ from the starting point of the search were eliminated. The resulting sample size of suitable search patterns to use in the current analysis thus amounted to 10 search runs. Fig. $2 \mathrm{~b}$ shows the combined search paths of the ten runs which produced the expected radially symmetrical distribution.

The search data thus began as sets of $(x, y)$ coordinates relative to the release point (which was also the correct nest position). In the next step, searches were interpolated with cubic splines using the Matlab 'spline' command to allow total path length to be calculated. The paths were resampled using the simulation time step, retaining the individual speeds of the different ants. Mean walking speed was $0.248 \mathrm{~ms}^{-1}$ with standard deviation 0.032 . The resampled paths were played back in the same manner as the spiral and Lévy searches except that no recording or executions errors were applied (i.e. $\sigma_{\text {exe }}^{2}=\sigma_{\text {rec }}^{2}=0.0$ ), since the searches must already contain the effects of the PI errors the ants were subject to. The real searches began without first having performed any preceding foraging excursion and are therefore expected to have a minimal initial PI error. To generate an initial error (thus simulating a preceding excursion) the initial PI error, $\mathbf{e}_{\mathbf{0}}$, was randomly sampled in the same manner as for the other search models, see Eq. (6), and the searches were replayed starting from $\hat{\mathbf{a}}_{\mathbf{0}}=\mathbf{t}$ and $\mathbf{a}_{\mathbf{0}}=\mathbf{t}-\mathbf{e}_{\mathbf{0}}$. Without some initial error the replayed ant searches, and indeed all the search models, must always start at the true nest position, and therefore immediately find the nest with high probability.

\subsection{Estimation of the error rate}

An estimate of the PI error rate in C. fortis was obtained from experiments where ants had walked different distances before beginning to search for the nest (Merkle et al., 2006b). Ants were trained to a feeder $5 \mathrm{~m}(n=51), 10 \mathrm{~m}(n=53)$ or $20 \mathrm{~m}(n=50)$ south of the nest. After at least a day of training ants were captured at the feeder, released in the remote test area (see above), and allowed to home and search for the nest under conditions where PI was the only viable navigation strategy (see Fig. 2c). Three hundred seconds of homing and search were recorded for the 5- and $10-\mathrm{m}$ ants and $600 \mathrm{~s}$ for the $20-\mathrm{m}$ ants. As described above (see Section 2.4), these trajectories were transformed into Cartesian coordinates. The median $x$ and $y$ coordinates of the entire search pattern where taken to indicate the ants' estimate of the nest position. The patterns usually consist of a small number of loops appearing to branch out from a central position, hence the median was selected as the averaging method to reduce the influence of individual loop directions (Merkle and Wehner, 2009b). Fig. 3 
shows the variance of the $x$ and $y$ coordinates as a function of the round-trip distance walked prior to search, along with $95 \%$ confidence intervals. The $x$ coordinate indicates position orthogonal to and the $y$ coordinate parallel to the nest-feeder direction. Hence $y$ variation arises from under- or over-running the correct homing

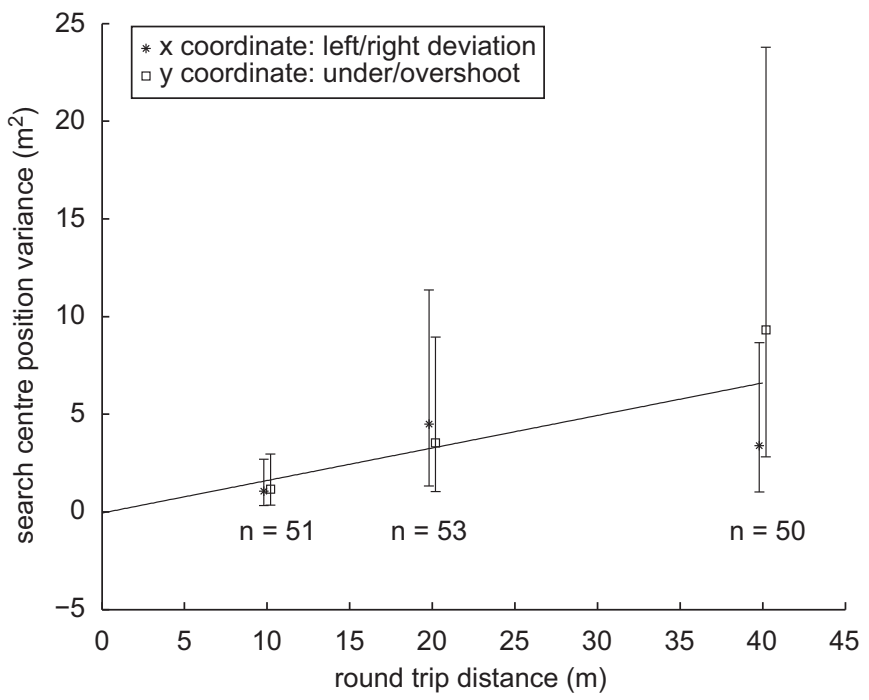

Fig. 3. Estimation of PI error rate. Plot shows the variance of the $x$ and $y$ coordinate of the search centre position as a function of the total distance walked prior to beginning to search for the nest, with $95 \%$ confidence intervals. A straight line was fitted to the six points yielding an error rate of $0.0416 \mathrm{~m}^{2} / \mathrm{s}$ assuming a walking speed of $0.25 \mathrm{~m} / \mathrm{s}$. For experimental details see Merkle et al. (2006b). distance, whereas $x$ variation arises from deviations to the left or right of the correct position. A straight-line was fitted to the six data points using the least-squares method. Assuming a mean walking speed of $0.25 \mathrm{~m} / \mathrm{s}$ the slope corresponds to a variance increasing at a rate of $\sigma_{\text {err }}^{2}=0.042 \mathrm{~m}^{2} / \mathrm{s}$. The fitted variance for a journey of zero length is $-0.015 \mathrm{~m}^{2}$, i.e. close to zero as assumed by the model. We prefer to treat this error rate estimate as more of an upper bound than an unbiased estimate for several reasons. Firstly, an ant may deviate from the geometrically expected search position deliberately, perhaps in order to search for memorised nest cues which are obviously absent in the test area. Secondly, positional error should, of course, be assumed to continue accruing during a PI-guided systematic search, but here we have fitted the straight line to the graph as if the positional error estimated from the whole search pattern were due only to the error accumulated during the pre-search portion of the journey. A more accurate method would also need to take account of the drift occurring during the search itself.

\subsection{Comparison of search methods}

Unless otherwise stated, all simulated searches were conducted using the following parameter values: $1 \mathrm{~s}$ per simulation timestep, search duration $n_{s}=300 \mathrm{~s}, 0.1 \mathrm{~m}$ per simulation distance unit (i.e. $100 \mathrm{PDF}$ values per square metre), animal speed $\lambda=0.25 \mathrm{~m} / \mathrm{s}$, maximum detection probability $\alpha=0.95$, detection range $\beta=0.2 \mathrm{~m}$, detection drop-off $\gamma=7$, pre-search foraging time $n_{0}=100 \mathrm{~s}$ (equivalent to a round trip to a feeder $12.5 \mathrm{~m}$ away assuming $0.25 \mathrm{~m} / \mathrm{s}$ walking speed), search failure cost $c_{f}=5$ resource units. In the absence of any relevant experimental data to assign the error rate between execution and recording error, for a given error rate, $\sigma_{\mathrm{err}}^{2}$, the execution and recording error rates
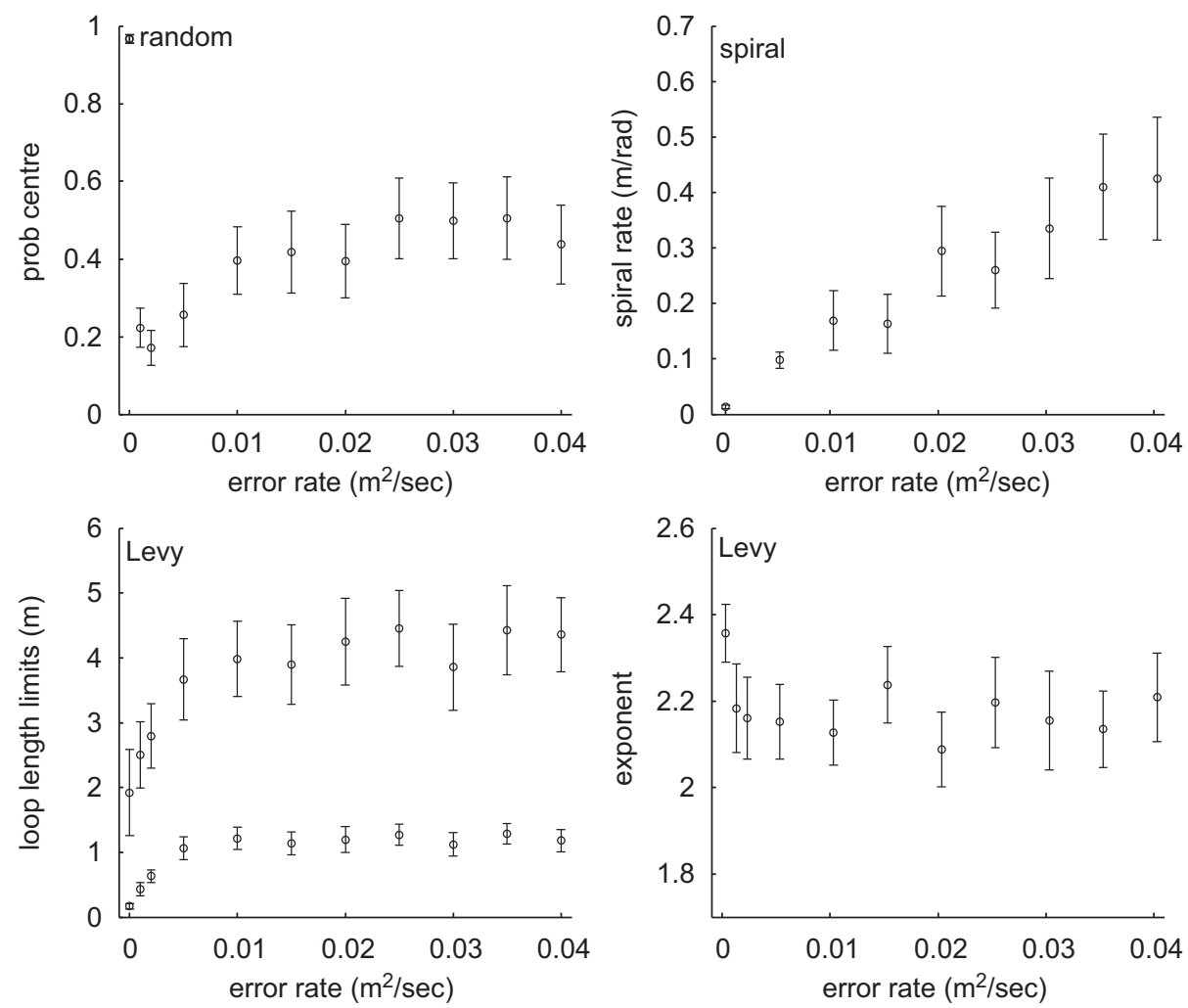

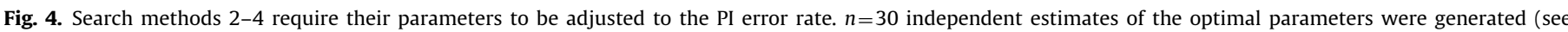

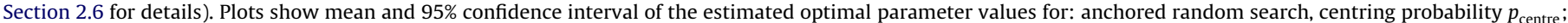
Archimedean spiral search, spiral rate parameter $c_{\text {spiral }}$; Lévy loop search, loop length parameters $l_{\min }$ and $l_{\max }$, and exponent parameter $b$. 
were assigned an equal share: $\sigma_{\text {exe }}^{2}=\sigma_{\text {rec }}^{2}=\sigma_{\text {err }}^{2} / 2$. There is insufficient experimental data available to accurately determine the detection function parameters, although a range as low as $2-3 \mathrm{~cm}$ would be consistent with observations of returning foragers passing close to the nest entrance without entering it (Merkle, personal observations). Unfortunately using a value this small would require a spatial resolution for the PDF that would make the Bayesian search method run extremely slowly. Instead we have chosen a larger value which allows the model to execute at a reasonable speed, allowing the elucidation of the search performance over a range of error rates.

In order to compare the search efficiency of the various models across a range of error rates, it is necessary to estimate the optimal parameter values for models $2-4$ as a function of the error rate. For each of a series of error rates between 0 and $0.04 \mathrm{~m}^{2} / \mathrm{s}$, optimal parameters were estimated as follows (see Fig. 4 for the error rates tested and the parameter values obtained).

For the two search methods requiring a single parameter to be optimised (i.e. $p_{\text {centre }}$ for anchored random search and $c_{\text {spiral }}$ for spiral search), a stratified random sample of 30 parameter values was generated by dividing the parameter range $[0,1]$ into 30 equal strata and picking one random value within each. The search performances were estimated by simulating 30 searches and taking the mean $\mu_{\max }$ for each parameter value. The value giving the highest search performance was retained as an estimate of the optimal parameter value. This whole procedure was repeated 30 times in total, giving 30 independent estimates of the optimal parameter value. The mean of the 30 estimates was used as the optimal parameter value for the error rate being tested. This averaging procedure was found to be more robust than simply using the parameter value which gave the highest resource accumulation rate owing to the significant influence of noise on performance, especially for large PI error rates. Plots of the performance against parameter values for each error rate (data not shown) did not give any indication that the performance curves were bimodal, and therefore the average of multiple estimates ought to give a good approximation of the true optimum.

For the Lévy loop search three parameters require optimisation. It would be too computationally expensive to stratify each parameter into 30 bands and sample from all $30^{3}$ regions of parameter space, therefore a modified procedure was used. Firstly, rough estimates of the optimal parameter values were generated using a simplified version of the procedure for the single parameter models: for each error rate 30 sets of the three parameters were generated using simple random sampling with uniform probabilities within the respective ranges $\left(l_{\min } \in\right.$ $\left.[0.001,2.0], l_{\max } \in\left[l_{\min }+0.1, l_{\min }+10\right], b \in[1,3]\right)$ instead of stratified random sampling. The rest of the rough-estimate procedure was the same as that just described for the single parameter optimisation. These rough estimates enabled the parameter ranges to be narrowed down somewhat to $l_{\min } \in[0.001,2.0]$, $l_{\max } \in\left[l_{\min }+0.1, l_{\min }+6\right], b \in[1.7,2.6]$. These ranges were then each stratified into 5 bands of equal width, giving $5^{3}=125$ regions of parameter space in total. Now the procedure was followed again: a stratified random sample of 125 parameter sets was chosen, picking one each from the 125 regions. Search performance was estimated by simulating 30 searches for each. The parameter set giving the best performance was taken as an estimate of the optimum parameter set. This sampling process was repeated 30 times in total giving 30 independent estimates of the optimum parameter set. The mean of each parameter across these 30 sets was taken to be the optimum.

The estimated optimum parameter values were plotted along with a $95 \%$ confidence interval against the error rate. Fig. 4 shows the mean and confidence interval for the estimated optimal parameter values for the three search methods.

\subsection{Implementation details}

The Bayesian model (model 1) defined in Section 2.3 was implemented in Matlab (Version 7.3.0.298, R2006b). Several refactorisations were made in the implementation which did not alter the behaviour of the model in anyway but allowed the code to execute significantly faster. In order to speed up the calculation of the dot product $\hat{s}_{k}=\mathbf{P}_{\mathbf{k}} \cdot \mathbf{D}\left(\hat{\mathbf{a}}_{\mathbf{k}}\right)$ the following changes were made. Column vector $\mathbf{P}_{\mathbf{k}}$ was implemented as a two-dimensional matrix, $\left\{\mathbf{P}_{\mathbf{k}}\right\}$, where position within the matrix corresponded to the spatial coordinate of $\mathbf{z}_{\mathbf{i}}$. Column vector function $\mathbf{D}\left(\hat{\mathbf{a}}_{\mathbf{k}}\right)$ was similarly implemented as a two-dimensional matrix, $\{\mathbf{D}\}$, where position within the matrix corresponded to the spatial coordinate of $\mathbf{z}_{\mathbf{i}}$. For notational convenience $\mathbf{D}$ was originally defined as a function of $\hat{\mathbf{a}}_{\mathbf{k}}$, but a more efficient implementation is to take advantage of the fact that the detection probabilities are a function of range, and simply translate around in space as the animal moves. Therefore to avoid having to recalculate $\{\mathbf{D}\}$ as $\hat{\mathbf{a}}_{\mathbf{k}}$ changed, the values were calculated once, corresponding to the case $\mathbf{D}\left(\mathbf{v}_{\mathbf{0}}\right)$ and the translation of the function in space was implemented by effectively translating $\left\{\mathbf{P}_{\mathbf{k}}\right\}$ relative to $\{\mathbf{D}\}$ as shown in Fig. 1 . In order to calculate the dot product $\hat{s}_{k}=\mathbf{P}_{\mathbf{k}} \cdot \mathbf{D}\left(\hat{\mathbf{a}}_{\mathbf{k}}\right)$ the correct region of overlap, if any, between the two matrices $\left\{\mathbf{P}_{\mathbf{k}}\right\}$ and $\{\mathbf{D}\}$ was deduced from the values of $\mathbf{t}$ and $\hat{\mathbf{a}}_{\mathbf{k}}$ as shown in Fig. 1. If there was no overlap $\hat{s}_{k}$ was assigned a value of zero, otherwise the dot product was

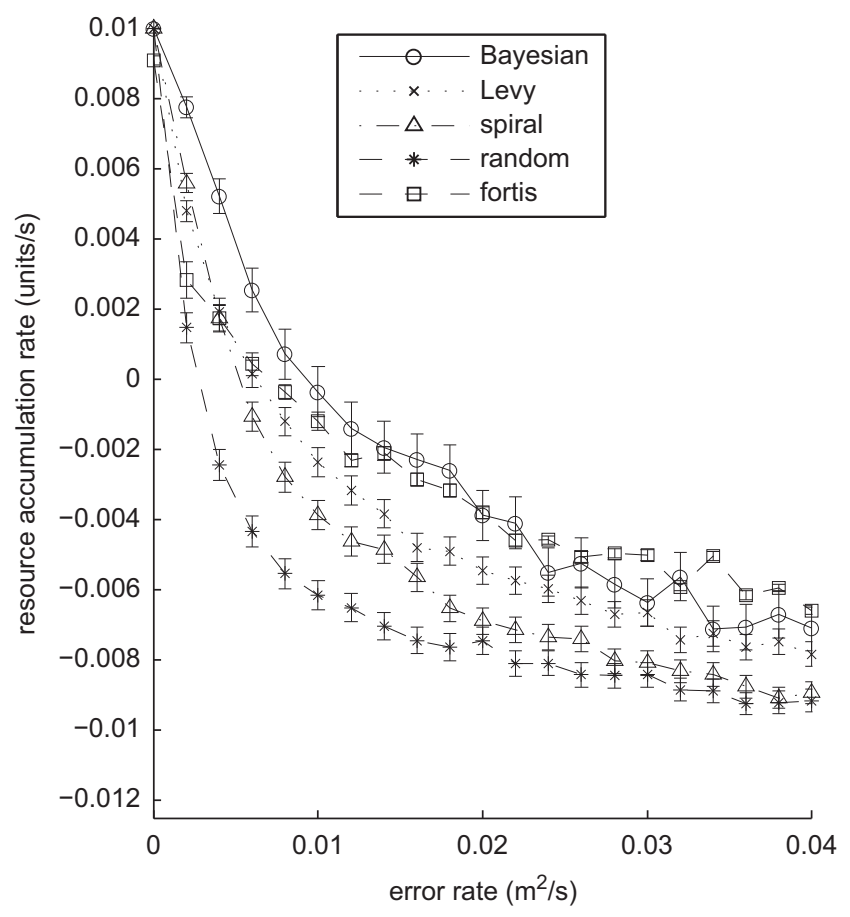

Fig. 5. Mean search performance plotted with $95 \%$ confidence intervals, as a function of error rate for the four search methods: Bayesian, Lévy loop, spiral, anchored random, and for recorded $C$. fortis search patterns. $n=500$ searches (Bayesian), $n=2000$ (Lévy, spiral, random), $n=10$ (ant search replay, each search replayed 100 times). For search methods Lévy, spiral and random, search parameters are set using the estimated optimal values previously obtained, see Fig 4. Search time $300 \mathrm{~s}$. Detection range $0.2 \mathrm{~m}$, animal walking speed $0.25 \mathrm{~m} \mathrm{~s}^{-1}$, mean ant walking speed $0.2479 \mathrm{~m} \mathrm{~s}^{-1}$ (with $\sigma=0.0315$ ). Resource accumulation rate $\left(\mu_{\max }\right)$ calculated assuming: foraging time $100 \mathrm{~s}$, failure cost five resource units. 
calculated within the region of overlap only, indicated by the shaded area, since at least one of the two matrix values must be zero outside this area. In this manner $\left\{\mathbf{P}_{\mathbf{k}}\right\}$ was treated as a PDF for the position of $\hat{\mathbf{a}}_{\mathbf{k}}-\mathbf{e}_{\mathbf{k}}=\mathbf{a}_{\mathbf{k}}$ and $\{\mathbf{D}\}$ defined the detection capability centred on position $\mathbf{t}$ as if the target were detecting the animal, but since detection is purely a function of range this gives
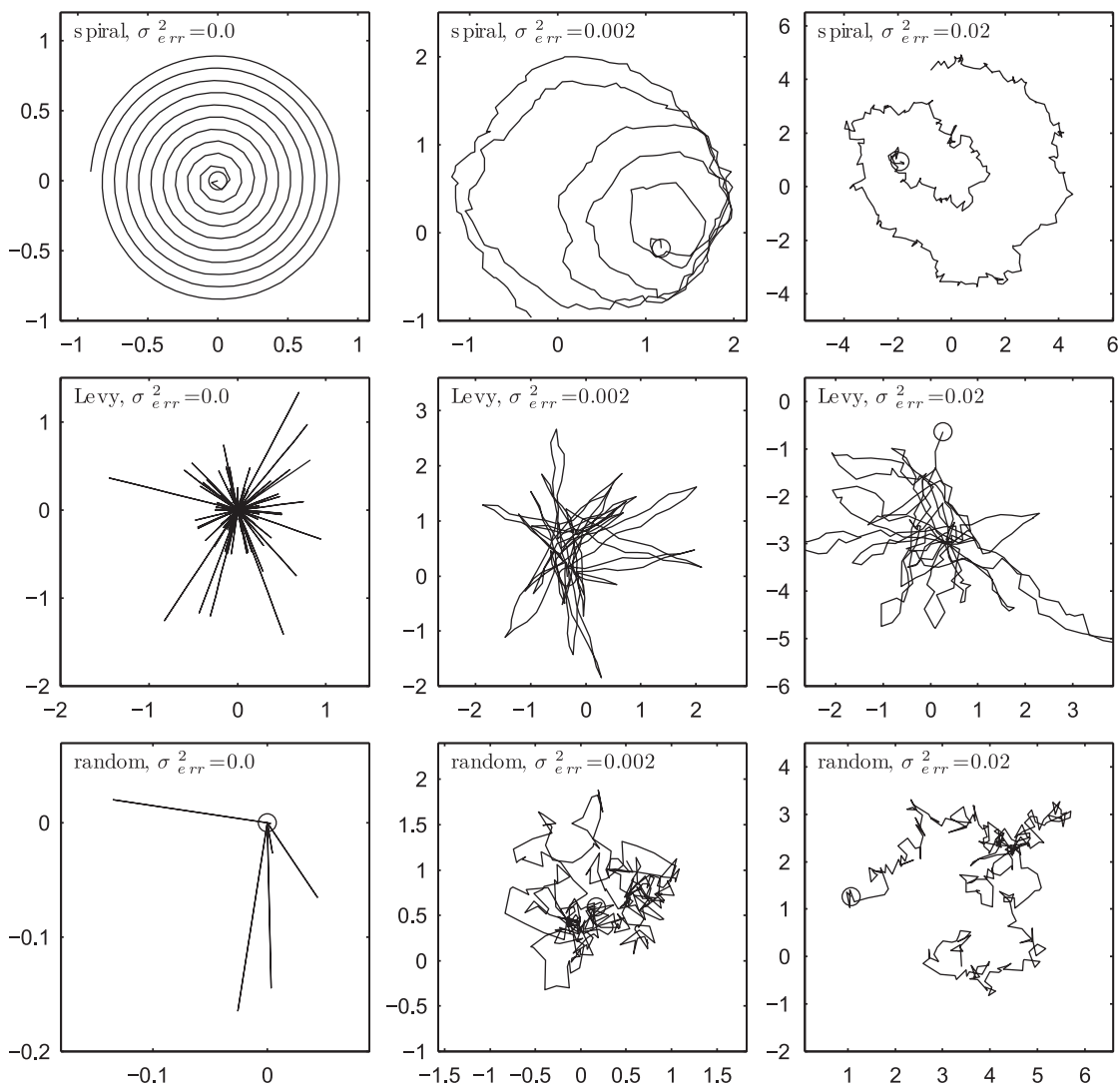

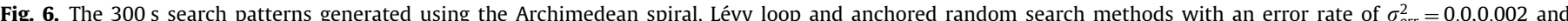

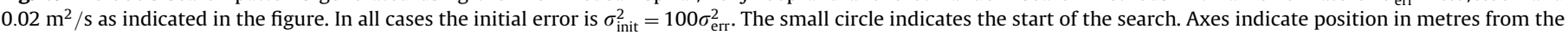
true target location.
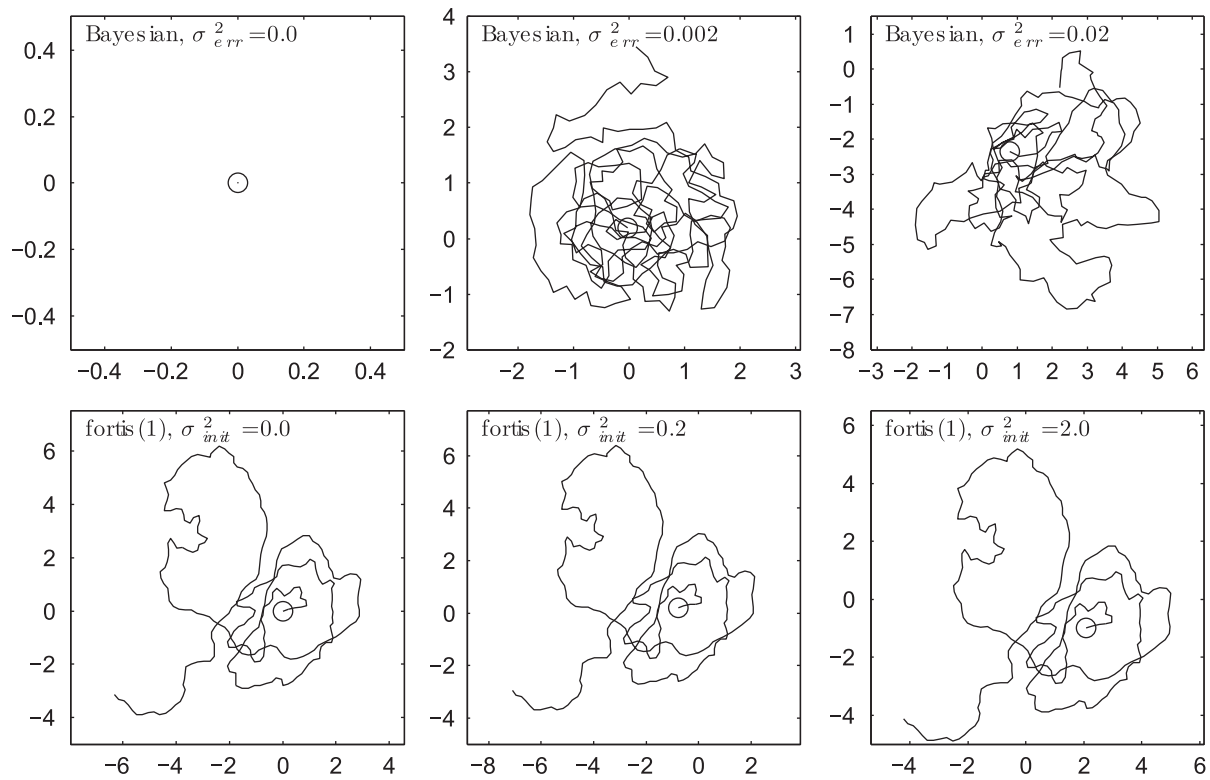

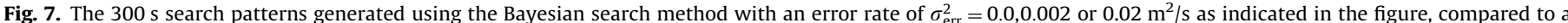

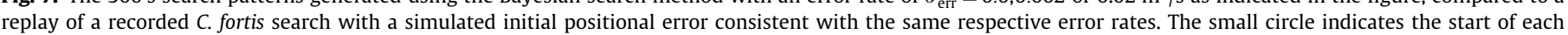

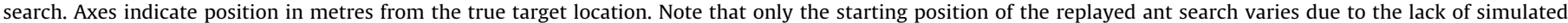
execution and recording errors (see Section 2.4). 
the same result as for the animal detecting the target. The indexing of elements in $\left\{\mathbf{P}_{\mathbf{k}}\right\}$ by spatial location must be mirrored for both axes, since the error $\mathbf{e}_{\mathbf{k}}$ is subtracted from $\hat{\mathbf{a}}_{\mathbf{k}}$ not added.

A second significant speed up of the model was achieved by avoiding the costly matrix multiplication $\mathbf{P}_{\mathbf{k}+\mathbf{1}}=\mathbf{T} \mathbf{Q}_{\mathbf{k}}$. Instead the two-dimensional matrix $\left\{\mathbf{Q}_{\mathbf{k}}\right\}$ (analogous to $\left\{\mathbf{P}_{\mathbf{k}}\right\}$ but for column vector $\mathbf{Q}_{\mathbf{k}}$ ) was used to calculate $\left\{\mathbf{P}_{\mathbf{k}+\mathbf{1}}\right\}$ by the convolution of the appropriate two dimensional Gaussian blur kernel, defined by $\sigma_{\text {err }}^{2}$, which was further sped up by implementation as two, linearly separated one-dimensional blur kernel operations.

\section{Results}

Fig. 5 shows resource accumulation rates, $\mu_{\max }$, with $95 \%$ confidence intervals, plotted against the error rate for all the search models where models 2-4 use parameter values optimised to the error rate. Given the large uncertainties in the correctness of the modelling assumptions and parameters, the quantitative values of the replayed $C$. fortis search resource accumulation rates should not be considered reliable. For example, under natural conditions a $C$. fortis ant can usually search for longer than 5 min before dying of exhaustion (but this time limit is imposed by our available data), may be able to make use of other homing strategies than PI under some circumstances and may find food closer to the nest than the distance here assumed etc. The four algorithmic search models $1-4$ are being compared under exactly the same conditions, and therefore, for the parameter values chosen, the relative performances are correct and meaningful. Firstly we will summarise the results of the search models $1-4$, then secondly compare these to the replayed ant searches.

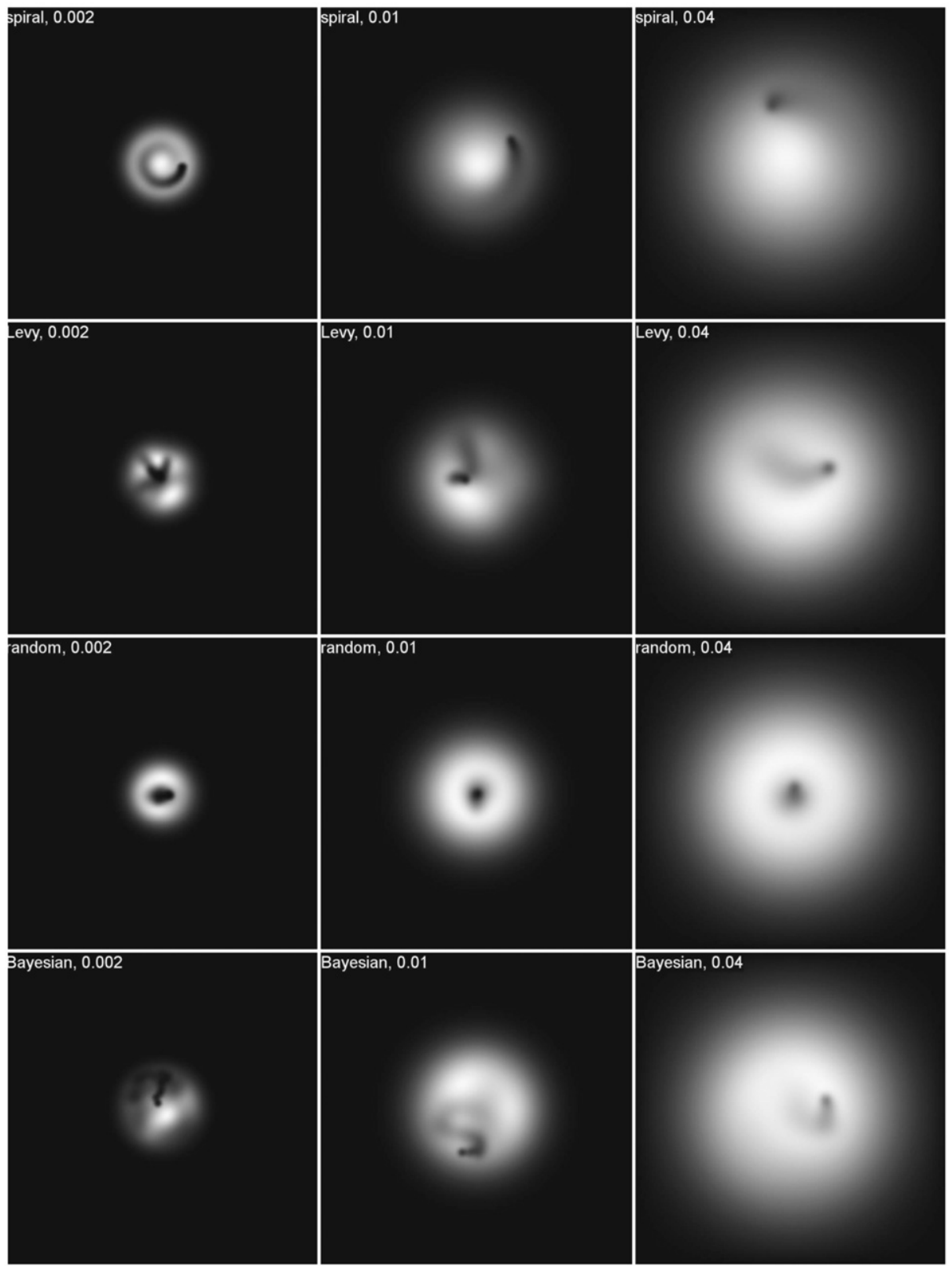

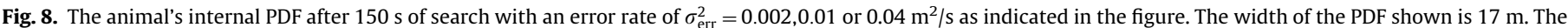
search method (also indicated in the figure) from top to bottom is: Archimedean spiral, Lévy loop, anchored random and Bayesian search. 
Fig. 5 shows, as expected, search performances start practically the same for an error rate $\sigma_{\text {err }}^{2}=0$, since this entails that the animal always starts directly on the target and need only move away moderately slowly in order the have multiple time steps

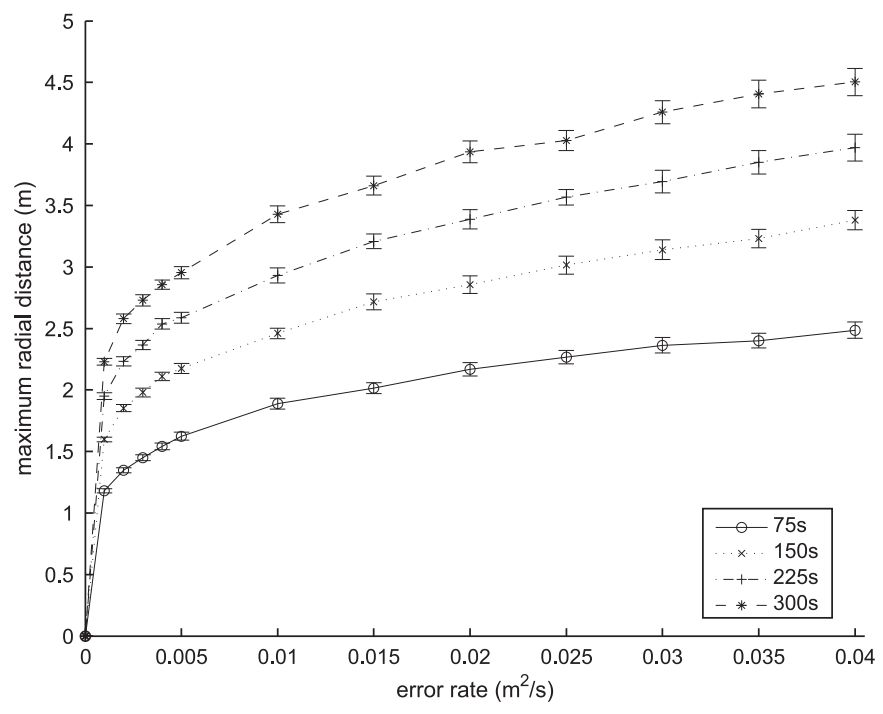

Fig. 9. Maximum spatial extent of the Bayesian search as a function of error rate. The distance is the maximum magnitude of the $\mathrm{HV}\left(\hat{a}_{k}\right)$ encountered over the first $75,150,225$ and $300 \mathrm{~s}$ of search respectively. The value shown is the mean of this value (plus 95\% confidence intervals) from a sample of $n=100$ searches. during which detection can occur with high probability. With increasing error rate the performances spread out to generally significantly different values, and then gradually converge towards equal performances for the highest error rates. Bayesian search significantly outperforms all other models for error rates below $\sigma_{\text {err }}^{2}=0.02 \mathrm{~m}^{2} / \mathrm{s}$ and its performance is never exceeded at any error rate. With a few interesting exceptions Lévy loops are second best, then spiral search, then anchored random search. The anchored random method is always worst or equal worst. The spiral method outperforms all the other non-Bayesian models at 0.002 , then declines in relative performance until at 0.01 it only exceeds the random search and at 0.03 begins to equal it. This is consistent with the expectation that this strategy is only suitable for low error conditions (since the efficiency of a spiral search which never returns to the centre is predicated on the assumption that the target will be detected on the first encounter), although it still outperforms the random search over a large range of error rates.

Direct comparison between search models 1-4 and the recorded ant searches is made difficult by the uncertainties in the parameter values best suited for modelling the ants' PI capabilities. The PI error rate was only estimated as an upper bound, implying only that the correct value is likely to be somewhere within the range tested. For technical reasons the nest detection range has been set above the value suggested by observations and in addition uncertainty remains as to which cues the ant uses to localise the nest and at what range. Only $300 \mathrm{~s}$ of search were recorded whereas ants can continue to search for much longer than this under natural conditions
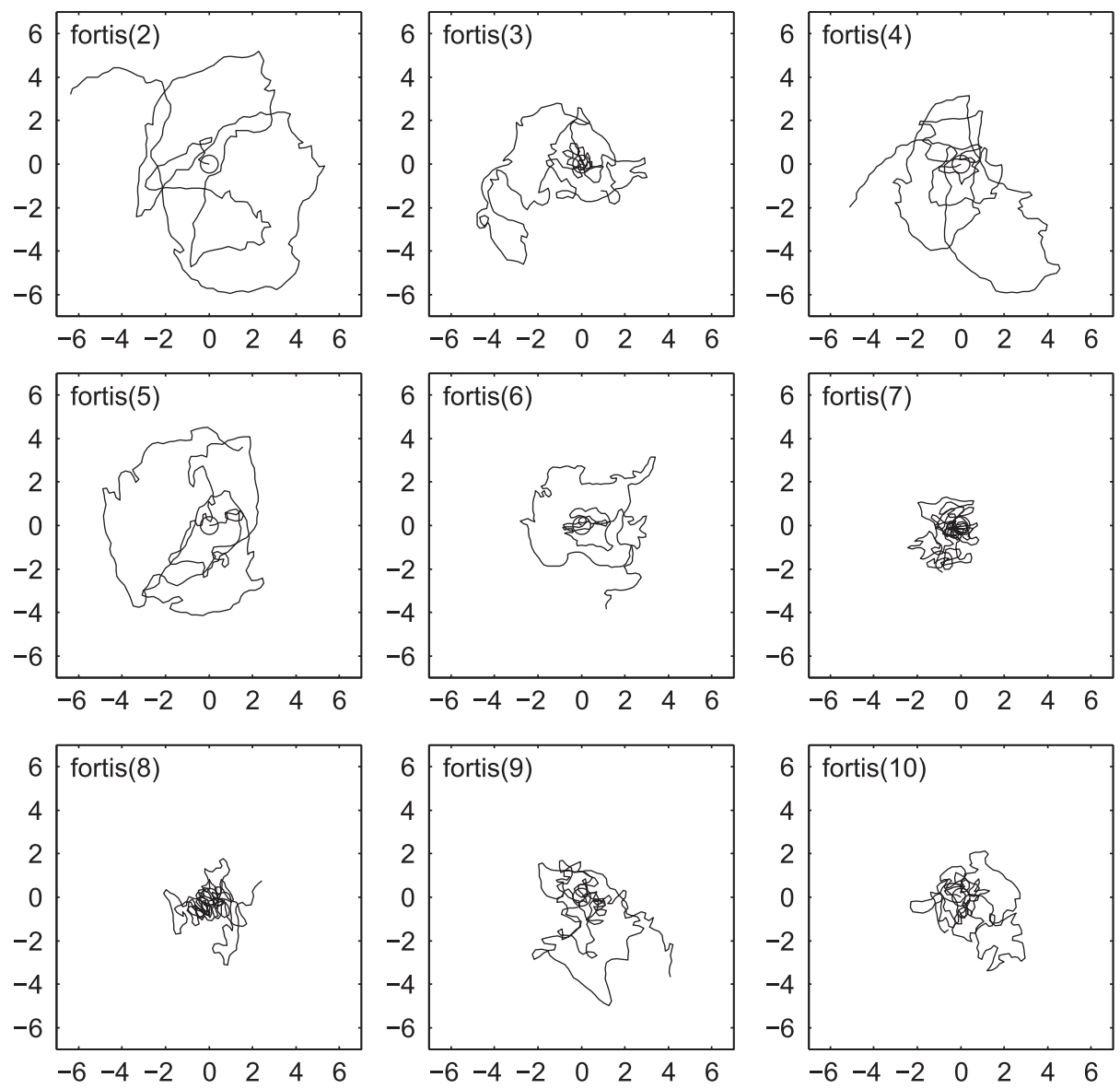

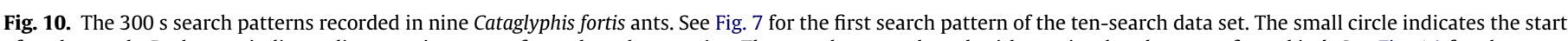

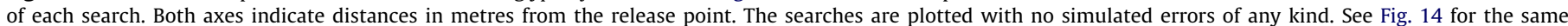
searches plotted as radial distances over time. 
(Müller and Wehner, 1994; Wehner and Srinivasan, 1981), therefore the simulation is likely to overestimate the value of $w_{f}$, the probability of search failure, and therefore exaggerate the influence of the search failure cost, $c_{f}$, (itself chosen without empirical data) on the resource accumulation rate. The pre-search foraging time is best kept to a minimum (approximately zero) for the purposes of recording ant search patterns, whereas the simulation requires that the animal begins searching with some positional error, otherwise the target will be found immediately. Therefore the simulation adds a random positional offset at the start of the ant search replay to simulate a preceding foraging period with associated PI error. This has the effect that the search efficiency is tested under conditions where the initial uncertainty is likely to be greater than that assumed by the ant when it began its search. An additional feature of the experimental paradigm used to record the search patterns (Merkle and Wehner, 2008) is that the ants were disturbed (captured and displaced) prior to beginning their searches. It is clear the ants registered some disturbance since, otherwise, when released they would have continued heading towards the presumed feeder position. Given the documented flexibility in the search patterns, it is plausible that any registered disturbance is capable of causing search pattern adaptation. Therefore, although we have endeavoured to minimise the PI error initially present when search began, we do not know how much positional uncertainty the ants have registered or how this effects the search pattern.

We therefore consider the calculated search performance of the recorded search patterns to be rough estimates, with significant scope for future refinement of model parameters. For error rates above 0.006 the estimated performance is very similar to the Bayesian method, being slightly inferior below 0.02 and slightly superior above 0.02 . Below 0.006 estimated performance is significantly below the Bayesian method (perhaps indicating the ants are experiencing an ongoing error rate larger than 0.006). If the nest detection capabilities of the ant have been poorly estimated then the Bayesian search will have an advantage since it automatically adapts to exploit the actual detection capabilities defined by the parameters whereas the ant searches will presumably be optimised to the actual nest detection capabilities of the ant, resulting in either over or under searching in the simulation.

Figs. 6 and 7 show three search patterns generated for each search method using error rates of $0.0,0.002$ and $0.02 \mathrm{~m}^{2} / \mathrm{s}$. For the case of the ant search replay the same search pattern is shown for all three error rates, in order to illustrate that the search pattern shape is not perturbed by any additional noise during the replay process (i.e. $\sigma_{\mathrm{err}}^{2}=0$ ). Since the search pattern shape must already incorporate the effects of errors the ant actually experienced, only an initial positional uncertainty $\sigma_{\text {init }}^{2}$ of equal magnitude to that applied to the other search models is used. Fig. 8 shows the animal's internal PDF after $150 \mathrm{~s}$ of search for three error rates of $0.002,0.01$ and $0.04 \mathrm{~m}^{2} / \mathrm{s}$ for each search method except the ant replay (since the PDF is not meaningful here). Fig. 8 clearly shows that for the spiral search the PDF after $150 \mathrm{~s}$ has a pronounced maximum at the centre, to which the animal never returns, indicating that it has a tendency to miss the nest and spiral out beyond it. Fig. 9 shows the automatic adaptation of the
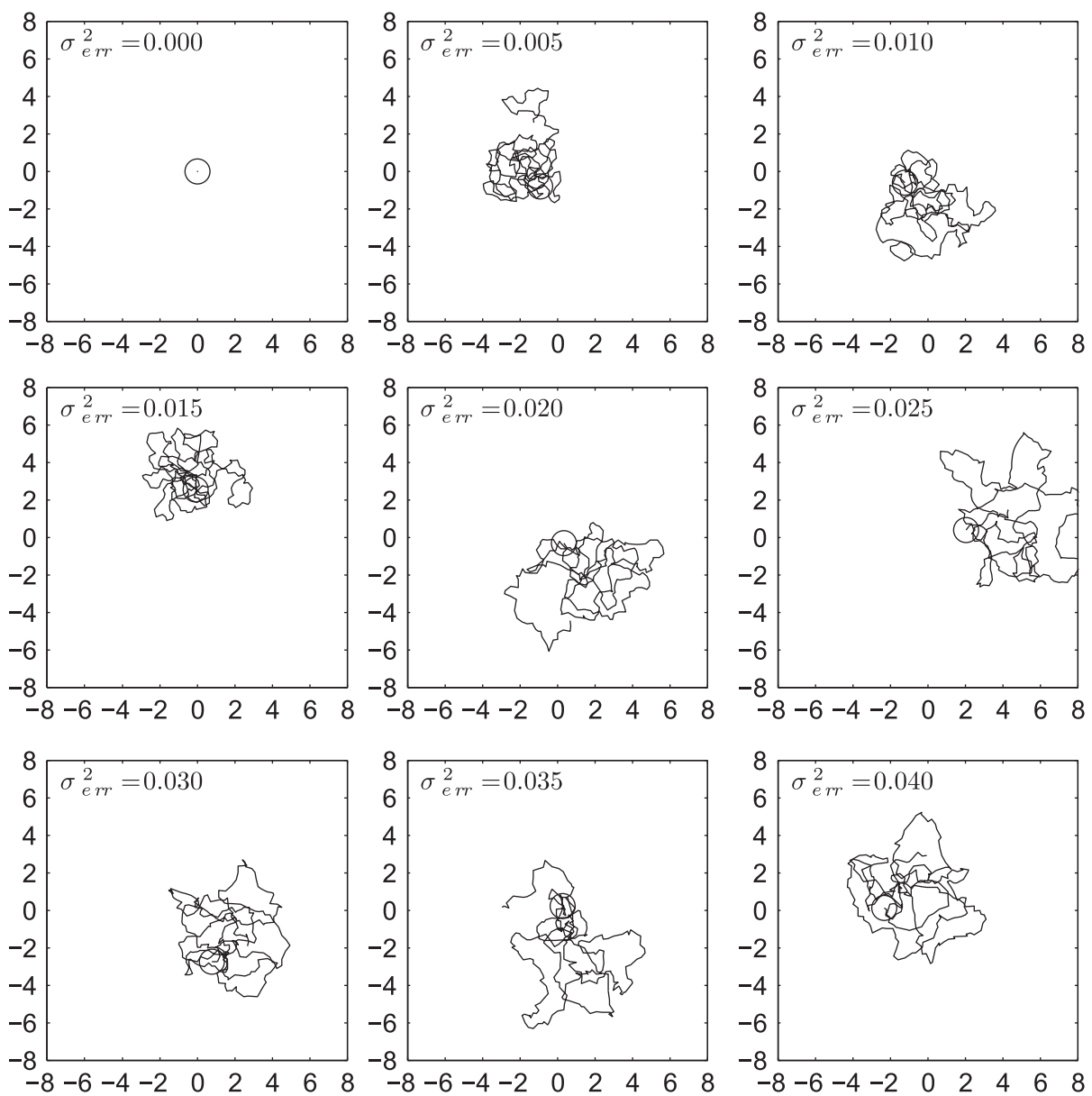

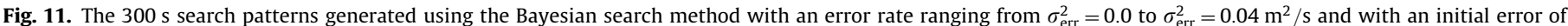
$\sigma_{\text {init }}^{2}=100 \sigma_{\text {err }}^{2}$. The small circle indicates the start of each search. Axes indicate position in metres from the true target location. 

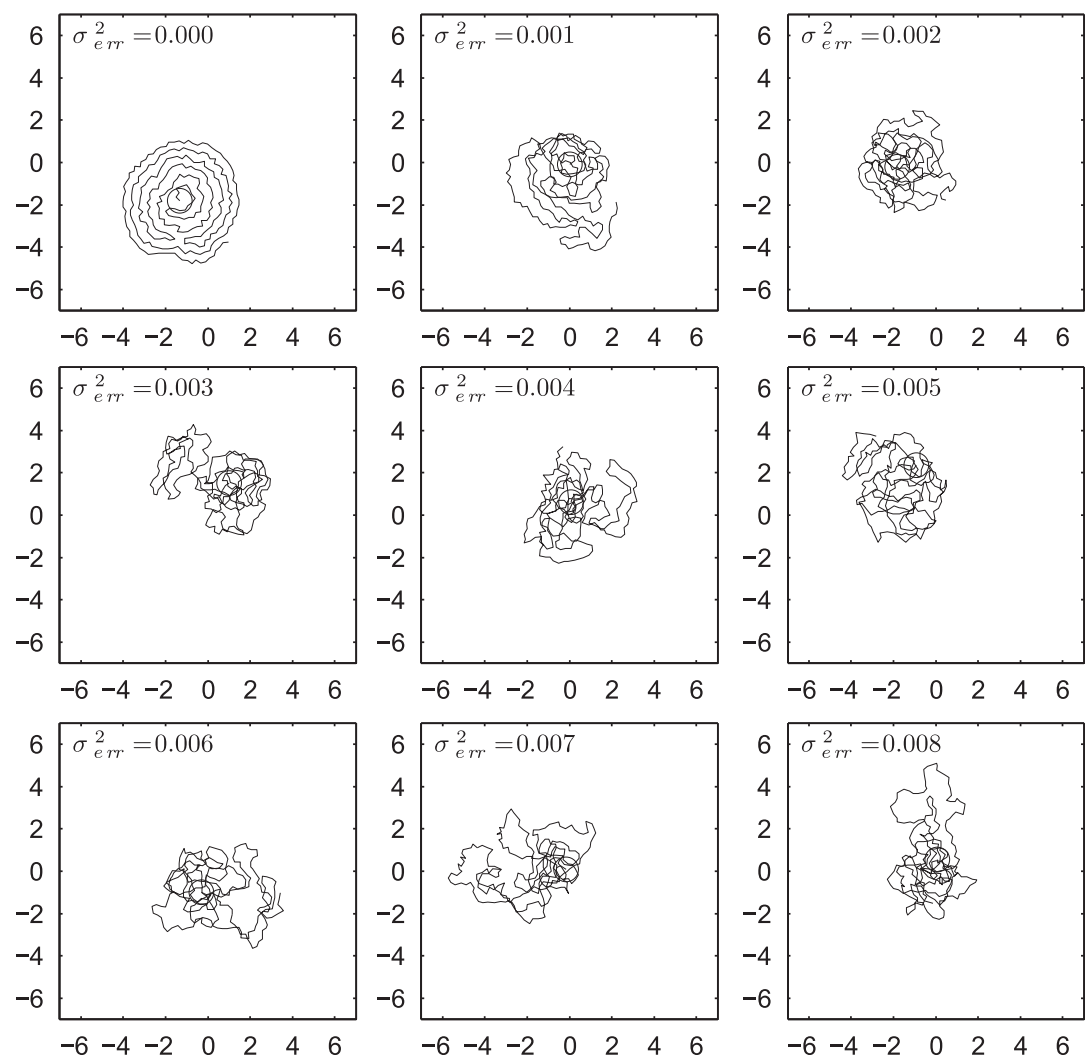

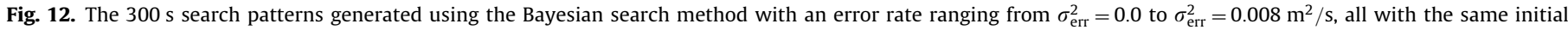

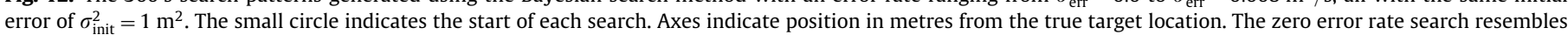
an Archimedean spiral with occasional changes in spiral direction.

Bayesian search to produce broader searches as the error rate increases. This is due to both a broader initial error PDF and to a faster spreading out of the error PDF during search. The plot shows the maximum value of $\left|\hat{\mathbf{a}}_{\mathbf{k}}\right|$ encountered over the first 75 , 150,225 and $300 \mathrm{~s}$ of search respectively.

To facilitate visual comparison of the search patterns produced by the Bayesian model and the ant, Fig. 10 shows the nine remaining ant search patterns (see Fig. 7 for the first) and Fig. 11 shows nine Bayesian model searches generated using zero initial positional uncertainty and an error rate of $\sigma_{\text {err }}^{2}=0.02$. Figs. 13 and 14 also show plots of the radial distance of the animal from the target location over time for all four models and for the recorded ant search patterns.

The Bayesian search has a number of interesting properties. It automatically adapts to the error rate and the initial uncertainty level without the need for explicit adaptation of any parameter, including approximating an Archimedean spiral for very low error rates (Fig. 12). Watching animations of the changing PDF a momentum-like property becomes apparent where the search tends to continue in the same direction despite the lack of any explicit orientation variable in the model, due to the place it has recently searched having the lowest probability values. The method has no long range planning since it looks only one timestep ahead and therefore cannot deliberately cross a low probability area to reach a more favourable location, but 'momentum' appears to help with this somewhat.

\section{Discussion}

A simple Bayesian search heuristic, based on maximising the probability of finding the target during the next timestep and employing Bayesian updating of a PDF of the PI error, has been shown suitable for generating target searches that resemble the systematic search patterns seen in C. fortis ants, and which automatically adapt to increases in positional uncertainty to perform wider search patterns. Since the method only considers one timestep ahead, it is not guaranteed to generate strictly optimal searches, but is easily able to outperform three simpler search heuristics based on the Archimedean spiral, Lévy loops and anchored random movements. A more complex model might plan several moves ahead, or employ a global measure of the reduction in positional uncertainty, such as the entropy of the error PDF (cf. Vergassola et al., 2007). However, the model already employs a costly high spatial resolution PDF, and as such may be considered a more elaborate system than many biological navigators are likely to possess. In this regard it might be useful to test whether similar models using a lower spatial resolution PDF can still produce efficient searches, or whether some simple movement pattern heuristic is capable of approaching the efficiency of the present model without using a PDF at all. Three existing models of $C$. fortis searching exist (Wehner and Srinivasan, 1981; Müller and Wehner, 1994; Vickerstaff and Cheung, 2010), as well as one for $H$. reaumuri (Alt, 1995), and are comparable to the three non-Bayesian search methods investigated in this account, in that they generate searches without the use of an explicit PDF. Using the quantitative search efficiency methods presented in the present account, these search models can now be systematically compared and their search efficiency measured under a range of conditions. Further experimental measurements of the PI error rate and nest detection capabilities of $C$. fortis would be beneficial, along with any data capable of testing whether our chosen measure of search efficiency, the mean resource accumulation rate, is biologically appropriate.

Quantitative methods for characterising and comparing search pattern shapes would be beneficial. One obvious possibility would 

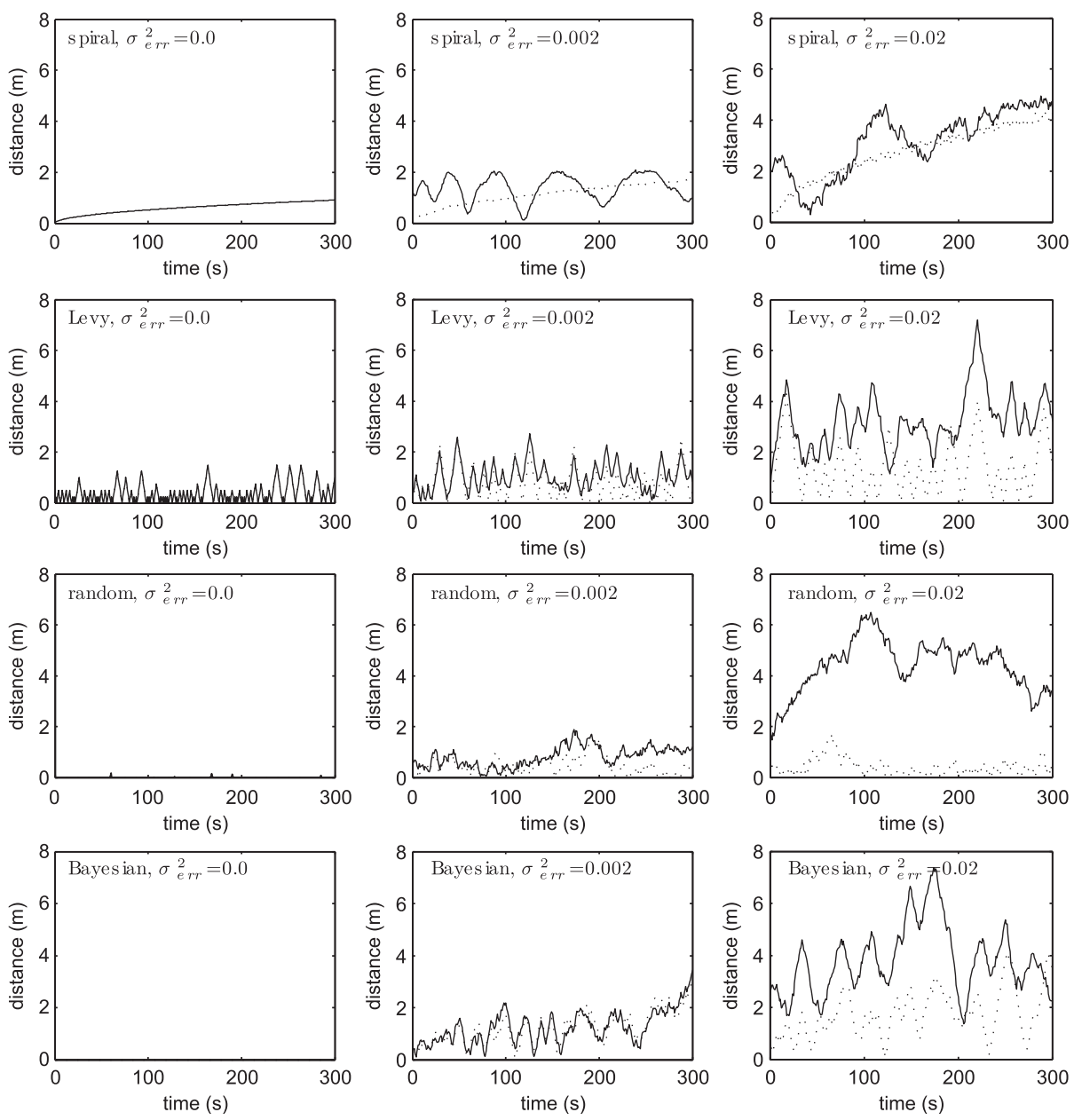

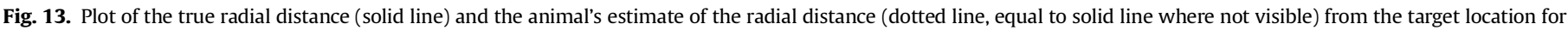
the Archimedean spiral, Lévy loop, anchored random and Bayesian search methods for three different error rates $\sigma_{\text {err }}^{2}=0.0,0.002$ and $0.02 \mathrm{~m}^{2} / \mathrm{s}$. In all cases $\sigma_{\text {init }}^{2}=100 \sigma_{\text {err }}^{2}$.

be to measure the time spent making radial versus orbital movements with respect to the central nest position. In such a scheme the Archimedean search represents the orbital extreme and the Lévy loop model the radial with the other models intermediate. A frequency domain analysis of the radial distance from the nest is another possible approach. If search pattern shapes can be characterised precisely enough perhaps a large sample of recorded search patterns can enable a statistical comparison with model-generated patterns, allowing the validation or falsification of models. This approach has the advantage of requiring only the search pattern shape of the animal under study, and does not need the direct measurement of the target detection range or PI error rate etc in the animal. The lessons learned from the search for Lévy flights in recordings of large scale movements (Humphries et al.,) may be relevant, such as awareness of the possibility that the data may represent two or more distinct strategies.

Hoffmann's (1983a) analysis of searching in the desert isopod $H$. reaumuri makes use of an optimal search plan calculated using search theory, based on the treatment of the problem as a stationary-target optimal search density problem (Stone, 1989). Such an optimal search plan is derived by assuming that searching effort can be freely divided between spatial locations without constraint. As such it can be considered as an upper bound on the realisable search performance, since a real searcher can only be at a single location at any one time (Alt, 1995), and must follow a definite path which it executes with some degree of uncontrollable, cumulative positional error. Given the conditions under which both the isopod and the ant are searching, the best orientation strategy available appears to be a compass-ASVR PI system (see Section 2.1). Given this assumption we have outlined our reasons for thinking the search problem is even then, in the general case, actually more akin to the much harder moving target, optimal searcher path problem (Stone, 1989), because (compass-ASVR) PI errors can be treated mathematically in much the same way as a randomly moving target. Whether the error rates encountered in nature are low enough for the simpler stationary-target optimal search density problem to describe the situation adequately remains to be demonstrated. If the animal lacks a compass-ASVR PI system then, presumably, the discrepancy between the ideal search plan and the animal's ability to approximate it will degrade even more rapidly as the level of noise increases and as the search lengthens owing to the inevitable drift between the reference frame of the search plan and the animal's actual position.

\section{Acknowledgments}

We wish to thank Allen Cheung and Wiebke Ebeling for help in the preparation of the paper. T.M. is grateful to Rüdiger Wehner for allowing him to collect the behavioural data at his Cataglyphis field station in Tunisia. T.M. was partly supported by the ARC Centre of Excellence in Vision Science and the ANU Centre for Visual Sciences. R.J.V. was partly supported by New Zealand Marsden Fund Grant UOC0507. 

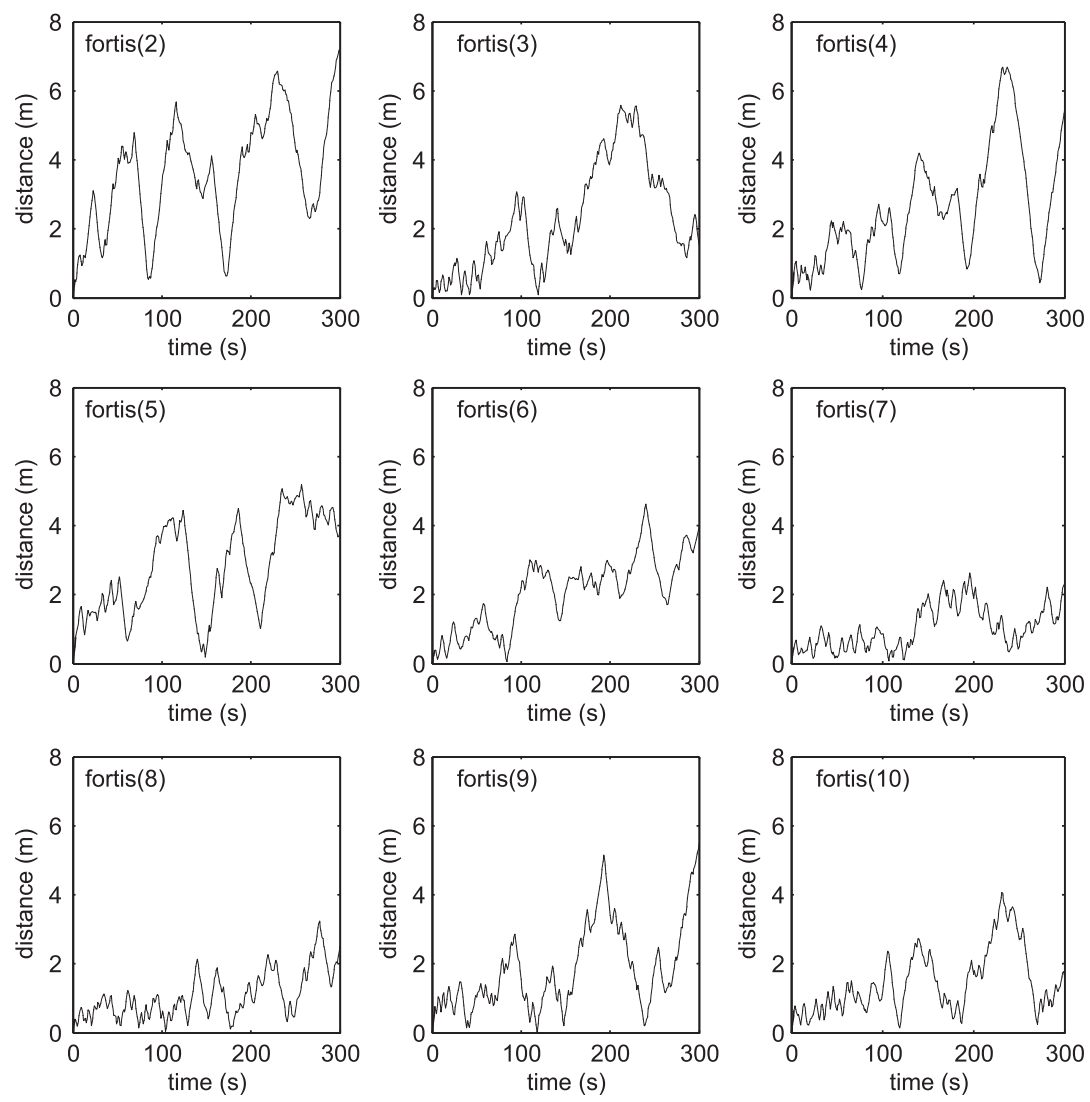

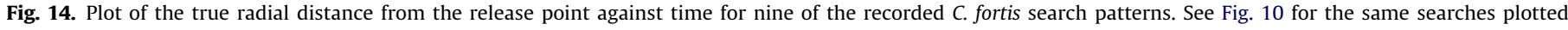
spatially.

\section{References}

Alt, W., 1995. Elements of a systematic search in animal behavior and model simulations. Biosystems 34, 11-26.

Antonsen, N., 1995. MbasaSoft GEDIT for Windows. v. 2.5. Technical report, Zürich: Institute of Zoology, University of Zürich.

Benkoski, S.J., Monticino, M.G., Weisinger, J.R., 1991. A survey of the search theory literature. Nav. Res. Log. 38, 469-494.

Cartwright, B.A., Collett, T.S., 1987. Landmark maps for honeybees. Biol. Cybern. 57, 85-93.

Cheng, K., Shettleworth, S.J., Huttenlocher, J., Rieser, J.J., 2007. Bayesian integration of spatial information. Psychol. Bull. 133, 625-637.

Cheung, A., Vickerstaff, R.J., 2010. Finding the way with a noisy brain. PLoS Comput. Biol. 6 (11), e1000992.

Cheung, A., Zhang, S., Stricker, C., Srinivasan, M.V., 2007. Animal navigation: the difficulty of moving in a straight line. Biol. Cybern. 97, 47-61.

Cheung, A., Zhang, S.W., Stricker, C., Srinivasan, M.V., 2008. Animal navigation: general properties of directed walks. Biol. Cybern. 99, 197-217.

Collett, M., Collett, T.S., 2000. How do insects use path integration for their navigation? Biol. Cybern. 83, 245-259.

Collett, M., Collett, T.S., 2006. Insect navigation: no map at the end of the trail? Curr. Biol. 16, R48-R51.

Collett, M., Collett, T.S., 2009. Local and global navigational coordinate systems in desert ants. J. Exp. Biol. 212, 901-905.

Cruse, H., Wehner, R., 2011. No need for a cognitive map: decentralized memory for insect navigation. PLoS Comput. Biol. 7 (3), e1002009.

Delcomyn, F., Usherwood, P.N.R., 1973. Motor activity during walking in the cockroach Periplaneta americana. I. Free walking. J. Exp. Biol. 59, 629-642.

Dell, R.F., Eagle, J.N., 1996. Using multiple searchers in constrained-path movingtarget search problems. Nav. Res. Log. 43, 463-480.

Dillier, F.X., Wehner, R., 2004. Spatio-temporal patterns of colony distribution in monodomous and polydomous species of North African desert ants, genus Cataglyphis. Insect. Soc. 51, 186-196.

Durier, V., Rivault, C., 1999. Path integration in cockroach larvae, Blattella germanica (L.) (Insect : Dictyoptera): direction and distance estimation. Learn. Behav. 27, 108-118.

Ellison, A.M., 2004. Bayesian inference in ecology. Ecol. Lett. 7, 509-520.

Etienne, A.S., Jeffery, K.J., 2004. Path integration in mammals. Hippocampus 14 (2), 180-192.

Fourcassie, V., Traniello, J.F.A., 1994. Food searching behaviour in the ant Formica schaufussi (Hymenoptera, Formicidae): response of naive foragers to protein and carbohydrate food. Anim. Behav. 48, 69-79.
Frost, J.R., 1999. Principles of search theory. Part I: Detection. Response 17, 1-7. Gallistel, C.R., 1990. The Organisation of Learning. MIT Press.

Harkness, R.D., Maroudas, N.G., 1985. Central place foraging by an ant (Cataglyphis bicolor Fab.): a model of searching. Anim. Behav. 33, 916-928.

Harkness, R.D., Wehner, R., 1977. Cataglyphis. Endeavour 11, 115-121.

Hartmann, G., Wehner, R., 1995. The ant's path integration system: a neural architecture. Biol. Cybern. 73, 483-497.

Hoffmann, G., 1983a. The search behavior of the desert isopod Hemilepistus reaumuri as compared with a systematic search. Behav. Ecol. Sociobiol. 13, 93-106.

Hoffmann, G., 1983b. The random elements in the systematic search behavior of the desert isopod Hemilepistus reaumuri. Behav. Ecol. Sociobiol. 13, 81-92.

Humphries, N.E., Queiroz, N., Dyer, J.R.M., Pade, N.G., Musyl, M.K., Schaefer, K.M., Fuller, D.W., Brunnschweiler, J.M., Doyle, T.K., Houghton, J.D.R., Hays, G.C., Jones, C.S., Noble, L.R., Wearmouth, V.J., Southall, E.J., Sims, D.W., 2010. Environmental context explains Lévy and Brownian movement patterns of marine predators. Nature 465, 1066-1069. 〈http://dx.doi.org/10.1038/nature09116〉.

Knaden, M., Wehner, R., 2006. Ant navigation: resetting the path integrator. J. Exp. Biol. 209, 26-31.

Lambrinos, D., Möller, R., Labhart, T., Pfeifer, R., Wehner, R., 2000. A mobile robot employing insect strategies for navigation. Robotics Auton. Syst. 30, 39-64.

Merkle, T., 2009. Surface structure helps desert ants return to known feeding sites. Commun. Integr. Biol. 2, 27-28.

Merkle, T., Wehner, R., 2008. Landmark guidance and vector navigation in outbound desert ants. J. Exp. Biol. 211, 3370-3377.

Merkle, T., Wehner, R., 2009a. Repeated training does not improve the path integrator in desert ants. Behav. Ecol. Sociobiol. 63, 391-402.

Merkle, T., Wehner, R., 2009b. How flexible is the systematic search behaviour of desert ants? Anim. Behav. 77, 1051-1056.

Merkle, T., Wehner, R., 2010. Desert ants use foraging distance to adapt the nest search to the uncertainty of the path integrator. Behav. Ecol. 21, 349-355.

Merkle, T., Rost, M., Alt, W., 2006a. Egocentric path integration models and their application to desert arthropods. J. Theor. Biol. 240, 385-399.

Merkle, T., Knaden, M., Wehner, R., 2006b. Uncertainty about nest position influences systematic search strategies in desert ants. J. Exp. Biol. 209, 3545-3549.

Mittelstaedt, H., Mittelstaedt, M.-L., 1982. Homing by path integration. In: Papi, F., Wallraff, H.G. (Eds.), Avian Navigation. Springer, pp. 290-297.

Müller, M., Wehner, R., 1988. Path integration in desert ants, Cataglyphis fortis. Proc. Natl. Acad. Sci. USA 85, 5287-5290.

Müller, M., Wehner, R., 1994. The hidden spiral: systematic search and path integration in desert ants, Cataglyphis fortis. J. Comp. Physiol. A 175, $525-530$. 
Redish, A.D., 1999. Beyond the cognitive map. From place cells to episodic memory. MIT Press.

Reynolds, A.M., 2008. Optimal random levy-loop searching: new insights into the searching behaviours of central-place foragers. Europhys. Lett. 82, 20001.

Richardson, H.R., Stone, L.D., 1971. Operations analysis during the underwater search for Scorpion. Nav. Res. Logist. Q. 18, 141-157.

Schmid-Hempel, P., Schmid-Hempel, R., 1984. Life duration and turnover of foragers in the ant Cataglyphis bicolor (Hymenoptera, Formicidae). Insect. Soc. $31,345-360$.

Seidl, T., Wehner, R., 2006. Visual and tactile learning of ground structures in desert ants. J. Exp. Biol. 209, 3336-3344

Stone, L.D., 1989. What's happened in search theory since the 1975 Lanchester prize? Oper. Res. 37, 501-506.

Tolman, E.C., 1948. Cognitive maps in rats and men. Psychol. Rev. 55, 189-208.

Vergassola, M., Villermaux, E., Shraiman, B.I., 2007. Infotaxis as a strategy for searching without gradients. Nature 445, 406-409.

Vickerstaff, R.J., Cheung, A., 2010. Which coordinate system for modelling path integration? J. Theor. Biol. 263, 242-261.
Vickerstaff, R.J., Di Paolo, E.A., 2005. Evolving neural models of path integration. J. Exp. Biol. 208, 3349-3366.

Wehner, R., 2003. Desert ant navigation: how miniature brains solve complex tasks. J. Comp. Physiol. A 189, 579-588.

Wehner, R., Müller, M., 2006. The significance of direct sunlight and polarized skylight in the ant's celestial system of navigation. Proc. Natl. Acad. Sci. USA 103, 12575-12579.

Wehner, R., Srinivasan, M.V., 1981. Searching behaviour in desert ants, genus Cataglyphis (Formicidae, Hymenoptera). J. Comp. Physiol. 142, 315-338.

Wehner, R., Srinivasan, M.V., 2003. The neurobiology of spatial behaviour. In: Jeffery, K.J. (Ed.), Path Integration in Insects. Oxford University Press, pp. $11-30$

Wehner, R., Wehner, S., 1986. Path integration in desert ants. approaching a longstanding puzzle in insect navigation. Monit. Zool. Ital. 20, 309-331.

Wehner, R., Wehner, S., 1990. Insect navigation: use of maps or ariadne's thread. Ethol. Ecol. Evol. 2, 27-48.

Wehner, R., Gallizzi, K., Frei, C., Vesely, M., 2002. Calibration processes in desert ants navigation: vector courses and systematic search. J. Comp. Physiol. A 188, 683-693. 